\title{
NEW FAMILIES OF WEIGHTED SUM FORMULAS FOR MULTIPLE ZETA VALUES
}

\author{
HAIPING YUAN AND JIANQIANG ZHAO
}

\begin{abstract}
In this paper we shall use the generating functions and the double shuffle relations satisfied by the multiple zeta values to derive some new families of identities of these values.
\end{abstract}

1. Introduction. In recent years, there is a flux of research on the multiple zeta functions and their special values due to their deep connections with many branches of mathematics and physics. For any positive integer $d$ (called the depth) and $s_{1}, \ldots, s_{d}$ with $s_{1}>1$ the multiple zeta values (MZVs) are defined by

$$
\zeta\left(s_{1}, \ldots, s_{d}\right)=\sum_{k_{1}>\cdots>k_{d}>0} \frac{1}{k_{1}^{s_{1}} \cdots k_{d}^{s_{d}}} .
$$

These values are easily seen to satisfy the so called stuffle relation. For example,

$$
\zeta\left(s_{1}\right) \zeta\left(s_{2}\right)=\zeta\left(s_{1}, s_{2}\right)+\zeta\left(s_{2}, s_{1}\right)+\zeta\left(s_{1}+s_{2}\right) .
$$

Euler [2] first studied the depth two case and obtained the following decomposition formula by using partial fraction techniques: for all $s_{1}, s_{2} \geq 2$, one has

$$
\zeta\left(s_{1}\right) \zeta\left(s_{2}\right)=\sum_{\substack{t_{1} \geq 2, t_{2} \geq 1 \\
t_{1}+t_{2}=s_{1}+s_{2}}}\left\{\left[\begin{array}{l}
t_{1} \\
s_{1}
\end{array}\right]+\left[\begin{array}{l}
t_{2} \\
s_{2}
\end{array}\right]\right\} \zeta\left(t_{1}, t_{2}\right) .
$$

2010 AMS Mathematics subject classification. Primary 05A15, 11M32.

Keywords and phrases. Multiple zeta values, generating functions, double shuffle relations.

The first author is partially supported by a summer research grant from York College of Pennsylvania and the second author is partially supported by NSF DMS1162116.

Received by the editors on February 3, 2013, and in revised form on December 2, 2013. 
Here, and in the rest of the paper, we will use the notation $\left[\begin{array}{l}a \\ b\end{array}\right]$ for $\left(\begin{array}{c}a-1 \\ b-1\end{array}\right)$ to save space. Although he did not consider the divergence problem, his approach has been made rigorous using modern techniques of regularization. Similar to formula (1.2), one can show that, for all $s_{1}, s_{3} \geq 2$ and $s_{2} \geq 1$,

$$
\begin{aligned}
\zeta\left(s_{1}, s_{2}\right) \zeta\left(s_{3}\right)= & \sum_{\substack{t_{1} \geq 2, t_{2} \geq 1 \\
t_{1}+t_{2}=s_{1}+s_{3}}}\left[\begin{array}{l}
t_{1} \\
s_{3}
\end{array}\right] \zeta\left(t_{1}, t_{2}, s_{2}\right) \\
& +\sum_{\substack{t_{1} \geq 2, t_{2}, t_{3} \geq 1 \\
t_{1}+t_{2}+t_{3} \\
=s_{1}+s_{2}+s_{3}}}\left[\begin{array}{c}
t_{1} \\
s_{1}
\end{array}\right]\left\{\left(\begin{array}{c}
t_{2}-1 \\
s_{2}-t_{3}
\end{array}\right)+\left[\begin{array}{l}
t_{2} \\
s_{2}
\end{array}\right]\right\} \zeta\left(t_{1}, t_{2}, t_{3}\right) .
\end{aligned}
$$

In fact, nowadays this can be derived easily by the shuffle relations satisfied by the iterated integral expression of MZVs (see [11, page 510]). By combining the stuffle and the shuffle relations one can obtain the so called double shuffle relations (see [7] for details).

Let $d$ be any positive integer, and define

$$
G_{d}\left(x_{1}, \ldots, x_{d}\right)=\sum_{s_{1}, \ldots, s_{d} \in \mathbb{N}, s_{1}>1} x_{1}^{s_{1}-1} \cdots x_{d}^{s_{d}-1} \zeta\left(s_{1}, \ldots, s_{d}\right) .
$$

In [3], Gangl, Kaneko and Zagier used the double shuffle relations of (1.1) and (1.2) to derive the following equation

$$
\begin{array}{r}
G_{2}(x+y, x)+G_{2}(x+y, y)-G_{2}(x, y)-G_{2}(y, x) \\
=\frac{G_{1}(x)-G_{1}(y)}{x-y},
\end{array}
$$

and proved some families of MZV identities. Machide [9] generalized this to depth three case using the extended (also called regularized) double shuffle relations.

It is well known that, in order to get complete linear relations between MZVs, one should consider regularized double shuffle relations. For example, the weighted sum formula of Ohno and Zudilin [10] states 
that

$$
\sum_{\substack{j \geq 2, k \geq 1 \\ j+k=n}} 2^{j} \zeta(j, k)=(n+1) \zeta(n) .
$$

Later, Guo and Xie [5] generalized (1.6) to arbitrary depths using regularized double shuffle relations (they in fact also used the sum formula which is another consequence of the regularized double shuffle relations [7]).

In this paper, we shall use generating functions of MZVs (1.4) to reformulate double shuffle relations and derive some new identities of MZVs. Notice that we do not use the extended double shuffle relations, which make the computation a little easier. All the identities obtained in this way are therefore finite extended double shuffle relations in the sense of [7]. For example, we get the following interesting result as a corollary (see Corollary 3.3) in depth three:

$$
\begin{aligned}
\sum_{\substack{j \geq 2, k, l \geq 1 \\
j+k+l=n}} 2^{j-1} \zeta(j, k, l)+\sum_{\substack{j \geq 2, k \geq 1 \\
j+k=n-1}} 2^{j} \zeta(j, k, 1) \\
=n \zeta(n-1,1)+3 \zeta(n-2,2)+\zeta(2, n-2)+2 \zeta(n),
\end{aligned}
$$

for every positive integer $n \geq 2$. For a new result in depth four, please see Corollary 5.2.

2. Depth 2: Some new identities. In this section, we will derive some new identities of double zeta values using the generating function $G_{2}$. To begin with, we recall the famous sum formula essentially known to Euler [2]:

$$
\sum_{\substack{j \geq 2, k \geq 1 \\ j+k=n}} \zeta(j, k)=\zeta(n)
$$

Using generating functions, Granville [4] and Zagier proved the following generalization to arbitrary depth first conjectured by Moen (see [6]):

$$
\sum_{k_{1} \geq 2, k_{2}, \ldots, k_{d} \geq 1, k_{1}+\cdots+k_{d}=w} \zeta\left(k_{1}, k_{2}, \ldots, k_{d}\right)=\zeta(w) .
$$


Our first result provides a weighted sum formula similar to but different from (1.6).

Theorem 2.1. Let $n$ be a positive integer. Then, for all $n \geq 3$,

$$
\sum_{k=2}^{n-1} k \zeta(k, n-k)=\zeta(2, n-2)+2 \zeta(n)-(n-2) \zeta(n-1,1),
$$

and all $n \geq 4$,

$$
\begin{aligned}
\sum_{k=2}^{n-1} k^{2} \zeta(k, n-k) & =3 \zeta(2, n-2)+2 \zeta(3, n-3)+6 \zeta(n) \\
& -(2 n-6) \zeta(n-2,2)-n(n-2) \zeta(n-1,1)
\end{aligned}
$$

Proof. Taking the substitution $x \rightarrow x t$ and $y \rightarrow y t$ in (1.5) and comparing the coefficients of $t^{n-2}$, we get

$$
\begin{array}{r}
\sum_{k=2}^{n-1}\left[(x+y)^{k-1} x^{j-1}+(x+y)^{k-1} y^{j-1}-x^{k-1} y^{j-1}-y^{k-1} x^{j-1}\right] \zeta(k, j) \\
=\left(\frac{x^{n-1}-y^{n-1}}{x-y}\right) \zeta(n),
\end{array}
$$

where $j=n-k$. Differentiating this equation with respect to $x$, we have

$$
\begin{aligned}
& (2.5) \sum_{k=2}^{n-1}\left[(k-1)(x+y)^{k-2} x^{j-1}+(j-1)(x+y)^{k-1} x^{j-2}\right. \\
& \left.+(k-1)(x+y)^{k-2} y^{j-1}-(k-1) x^{k-2} y^{j-1}-(j-1) y^{k-1} x^{j-2}\right] \zeta(k, j) \\
& =\left(\frac{(n-1) x^{n-2}}{x-y}-\frac{x^{n-1}-y^{n-1}}{(x-y)^{2}}\right) \zeta(n) .
\end{aligned}
$$

Specializing to $(x, y)=(0,1)$, we find easily that

$$
(n-2) \zeta(n-1,1)+\sum_{k=2}^{n-1}(k-1) \zeta(k, n-k)-\zeta(2, n-2)=\zeta(n) .
$$

So (2.3) follows from the sum formula (2.1). 
Now multiplying $(2.5)$ by $x+y$, differentiating with respect to $x$, and then specializing to $(x, y)=(0,1)$, we get

$$
\begin{aligned}
(n-2)^{2} \zeta(n-1,1)+ & (2 n-6) \zeta(n-2,2) \\
& +\sum_{k=2}^{n-1}(k-1)^{2} \zeta(k, n-k) \\
& \quad-\zeta(2, n-2)-2 \zeta(3, n-3)=3 \zeta(n) .
\end{aligned}
$$

Hence, (2.4) quickly follows from (2.3) and the sum formula (2.1). This completes the proof of the theorem.

Remark 2.2. It is conceivable that, for every fixed positive integer $d$, a compact formula of $\sum_{k=2}^{n-1} k^{d} \zeta(k, n-k)$ can be obtained by differentiating (2.5) repeatedly, similar to what we have done in Theorem 2.1. However, it seems to be a difficult problem to find a general formula for all $d$.

3. Depth 3: Product of three Riemann zeta values. In the following, we use three different methods for computing the generating function of the product of three Riemann zeta values:

$$
\sum_{s_{1}, s_{2}, s_{3} \geq 2} x_{1}^{s_{1}-1} x_{2}^{s_{2}-1} x_{3}^{s_{3}-1} \zeta\left(s_{1}\right) \zeta\left(s_{2}\right) \zeta\left(s_{3}\right) .
$$

3.1. First method. Combining (1.2) and (1.3), we have

$$
\begin{aligned}
& \zeta\left(s_{1}\right) \zeta\left(s_{2}\right) \zeta\left(s_{3}\right)=\sum_{\substack{t_{1} \geq 2, t_{2} \geq 1 \\
t_{1}+t_{2}=s_{1}+s_{2}}}\left\{\left[\begin{array}{l}
t_{1} \\
s_{1}
\end{array}\right]+\left[\begin{array}{l}
t_{2} \\
s_{2}
\end{array}\right]\right\} \\
& \times\left\{\sum_{\substack{r_{1} \geq 2, r_{2} \geq 1 \\
r_{1}+r_{2}=t_{1}+s_{3}}}\left[\begin{array}{l}
r_{1} \\
s_{3}
\end{array}\right] \zeta\left(r_{1}, r_{2}, t_{2}\right)\right. \\
& \left.+\sum_{\substack{r_{1} \geq 2, r_{2}, r_{3} \geq 1 \\
r_{1}+r_{2}+r_{3} \\
=t_{1}+t_{2}+s_{3}}}\left[\begin{array}{c}
r_{1} \\
t_{1}
\end{array}\right]\left\{\left(\begin{array}{c}
r_{2}-1 \\
t_{2}-r_{3}
\end{array}\right)+\left[\begin{array}{l}
r_{2} \\
t_{2}
\end{array}\right]\right\} \zeta\left(r_{1}, r_{2}, r_{3}\right)\right\} \\
& =\sum_{\substack{t_{1} \geq 2, t_{2} \geq 1 \\
t_{1}+t_{2}=s_{1}+s_{2}}} \sum_{\substack{r_{1} \geq 2, r_{2} \geq 1 \\
r_{1}+r_{2}=t_{1}+s_{3}}}\left[\begin{array}{l}
t_{1} \\
s_{1}
\end{array}\right]\left[\begin{array}{l}
r_{1} \\
s_{3}
\end{array}\right] \zeta\left(r_{1}, r_{2}, t_{2}\right)
\end{aligned}
$$




$$
\begin{aligned}
& +\sum_{\substack{t_{1} \geq 2, t_{2} \geq 1 \\
t_{1}+t_{2}=s_{1}+s_{2}}} \sum_{\substack{r_{1} \geq 2, r_{2} \geq 1 \\
r_{1}+r_{2}=t_{1}+s_{3}}}\left[\begin{array}{l}
t_{2} \\
s_{2}
\end{array}\right]\left[\begin{array}{l}
r_{1} \\
s_{3}
\end{array}\right] \zeta\left(r_{1}, r_{2}, t_{2}\right) \\
& +\sum_{\substack{t_{1} \geq 2, t_{2} \geq 1 \\
t_{1}+t_{2}=s_{1}+s_{2}}} \sum_{\substack{r_{1} \geq 2, r_{2}, r_{3} \geq 1 \\
r_{1}+r_{2}+r_{3} \\
=t_{1}+t_{2}+s_{3}}}\left[\begin{array}{c}
t_{1} \\
s_{1}
\end{array}\right]\left[\begin{array}{l}
r_{1} \\
t_{1}
\end{array}\right]\left(\begin{array}{c}
r_{2}-1 \\
t_{2}-r_{3}
\end{array}\right) \zeta\left(r_{1}, r_{2}, r_{3}\right)
\end{aligned}
$$

$$
+\sum_{\substack{t_{1} \geq 2, t_{2} \geq 1 \\
t_{1}+t_{2}=s_{1}+s_{2}}} \sum_{\substack{r_{1} \geq 2, r_{2}, r_{3} \geq 1 \\
r_{1}+r_{2}+r_{3} \\
=t_{1}+t_{2}+s_{3}}}\left[\begin{array}{l}
t_{2} \\
s_{2}
\end{array}\right]\left[\begin{array}{l}
r_{1} \\
t_{1}
\end{array}\right]\left(\begin{array}{c}
r_{2}-1 \\
t_{2}-r_{3}
\end{array}\right) \zeta\left(r_{1}, r_{2}, r_{3}\right)
$$

$$
\begin{aligned}
& +\sum_{\substack{t_{1} \geq 2, t_{2} \geq 1 \\
t_{1}+t_{2}=s_{1}+s_{2}}} \sum_{\substack{r_{1} \geq 2, r_{2}, r_{3} \geq 1 \\
r_{1}+r_{2}+r_{3} \\
=t_{1}+t_{2}+s_{3}}}\left[\begin{array}{l}
t_{1} \\
s_{1}
\end{array}\right]\left[\begin{array}{l}
r_{1} \\
t_{1}
\end{array}\right]\left[\begin{array}{l}
r_{2} \\
t_{2}
\end{array}\right] \zeta\left(r_{1}, r_{2}, r_{3}\right) \\
& +\sum_{\substack{t_{1} \geq 2, t_{2} \geq 1 \\
t_{1}+t_{2}=s_{1}+s_{2}}} \sum_{\substack{r_{1} \geq 2, r_{2}, r_{3} \geq 1 \\
r_{1}+r_{2}+r_{3} \\
=t_{1}+t_{2}+s_{3}}}\left[\begin{array}{l}
t_{2} \\
s_{2}
\end{array}\right]\left[\begin{array}{l}
r_{1} \\
t_{1}
\end{array}\right]\left[\begin{array}{l}
r_{2} \\
t_{2}
\end{array}\right] \zeta\left(r_{1}, r_{2}, r_{3}\right) .
\end{aligned}
$$

We first treat (3.2)-(3.7) using binomial identities repeatedly to derive formulas involving the generating functions $G_{3}$. To save space, we only compute (3.2) in details and leave the others to the interested reader. Also we will use the shorthand $x_{i j}=x_{j}+x_{j}$ and $x_{i j k}=x_{j}+x_{j}+x_{k}$ in what follows. Now

$$
\begin{aligned}
& (3.2)=\sum_{\substack{s_{1}, s_{2}, s_{3}, t_{1} \geq 2, t_{2} \geq 1 \\
t_{1}+t_{2}=s_{1}+s_{2}}} \sum_{\substack{r_{1} \geq 2, r_{2} \geq 1 \\
r_{1}+r_{2}=t_{1}+s_{3}}}\left[\begin{array}{l}
t_{1} \\
s_{1}
\end{array}\right]\left[\begin{array}{l}
r_{1} \\
s_{3}
\end{array}\right] \\
& \cdot x_{1}^{s_{1}-1} x_{2}^{s_{2}-1} x_{3}^{s_{3}-1} \zeta\left(r_{1}, r_{2}, t_{2}\right) \\
& =\sum_{\substack{1, s_{2}, s_{3}, t_{1} \geq 2, t_{2} \geq 1 \\
t_{1}+t_{2}=s_{1}+s_{2}}} \sum_{\substack{r_{1} \geq 2, r_{2} \geq 1 \\
r_{1}+r_{2}=t_{1}+s_{3}}}\left[\begin{array}{l}
t_{1} \\
s_{1}
\end{array}\right]\left[\begin{array}{l}
r_{1} \\
s_{3}
\end{array}\right] \\
& \cdot x_{1}^{s_{1}-1} x_{2}^{t_{1}-s_{1}} x_{2}^{t_{2}-1} x_{3}^{s_{3}-1} \zeta\left(r_{1}, r_{2}, t_{2}\right) \\
& =\sum_{\substack{t_{1}, s_{3}, r_{1} \geq 2, t_{2}, r_{2} \geq 1 \\
r_{1}+r_{2}=t_{1}+s_{3}}}\left(x_{12}^{t_{1}-1}-x_{2}^{t_{1}-1}\right) x_{3}^{s_{3}-1}\left[\begin{array}{l}
r_{1} \\
s_{3}
\end{array}\right] \\
& \cdot x_{2}^{t_{2}-1} \zeta\left(r_{1}, r_{2}, t_{2}\right)
\end{aligned}
$$




$$
\begin{aligned}
& -\sum_{\substack{t_{1}, s_{3}, r_{1} \geq 2, r_{2} \geq 1 \\
r_{1}+r_{2}=t_{1}+s_{3}}} x_{1}^{t_{1}-1} x_{3}^{s_{3}-1}\left[\begin{array}{l}
r_{1} \\
s_{3}
\end{array}\right] \zeta\left(r_{1}, r_{2}, 1\right) \\
& =\sum_{\substack{t_{1}, s_{3}, r_{1} \geq 2, t_{2}, r_{2} \geq 1 \\
r_{1}+r_{2}=t_{1}+s_{3}}}\left(x_{12}^{r_{2}-1} x_{12}^{r_{1}-s_{3}}-x_{2}^{r_{2}-1} x_{2}^{r_{1}-s_{3}}\right) \\
& \cdot x_{3}^{s_{3}-1}\left[\begin{array}{l}
r_{1} \\
s_{3}
\end{array}\right] x_{2}^{t_{2}-1} \zeta\left(r_{1}, r_{2}, t_{2}\right) \\
& -\sum_{\substack{t_{1}, s_{3}, r_{1} \geq 2, r_{2} \geq 1 \\
r_{1}+r_{2}=t_{1}+s_{3}}} x_{1}^{r_{2}-1} x_{1}^{r_{1}-s_{3}} x_{3}^{s_{3}-1}\left[\begin{array}{c}
r_{1} \\
s_{3}
\end{array}\right] \zeta\left(r_{1}, r_{2}, 1\right) \\
& =\sum_{r_{1} \geq 1, t_{2}, r_{2} \geq 1}\left\{x_{12}^{r_{2}-1}\left(x_{123}^{r_{1}}-x_{12}^{r_{1}}\right)-x_{2}^{r_{2}-1}\left(x_{23}^{r_{1}}-x_{2}^{r_{1}}\right)\right\} \\
& \cdot x_{2}^{t_{2}-1} \zeta\left(r_{1}+1, r_{2}, t_{2}\right) \\
& -\sum_{\substack{t_{1}, s_{3}, r_{1} \geq 2, r_{2} \geq 1 \\
r_{1}+r_{2}=t_{1}+s_{3}}} x_{1}^{r_{2}-1}\left(x_{13}^{r_{1}-1}-x_{1}^{r_{1}-1}\right) \zeta\left(r_{1}, r_{2}, 1\right) \\
& +\sum_{r_{1} \geq 2} x_{3}^{r_{1}-1} \zeta\left(r_{1}, 1,1\right) \\
& =G_{3}\left(x_{123}, x_{12}, x_{2}\right)-G_{3}\left(x_{12}, x_{12}, x_{2}\right) \\
& -G_{3}\left(x_{23}, x_{2}, x_{2}\right)+G_{3}\left(x_{2}, x_{2}, x_{2}\right) \\
& -\sum_{\substack{t_{1}, s_{3}, r_{1} \geq 2, r_{2} \geq 1 \\
r_{1}+r_{2}=t_{1}+s_{3}}} x_{1}^{r_{2}-1}\left(x_{13}^{r_{1}-1}-x_{1}^{r_{1}-1}\right) \zeta\left(r_{1}, r_{2}, 1\right) \\
& +\sum_{r_{1} \geq 2} x_{3}^{r_{1}-1} \zeta\left(r_{1}, 1,1\right)
\end{aligned}
$$

Similarly, we find

$$
\begin{aligned}
(3.4)=G_{3}\left(x_{123}, x_{23}, x_{2}\right)-G_{3}\left(x_{23}, x_{23}, x_{2}\right) & \\
& -G_{3}\left(x_{12}, x_{2}, x_{2}\right)+G_{3}\left(x_{2}, x_{2}, x_{2}\right) \\
-\sum_{r_{1} \geq 2, r_{2} \geq 1}\left(x_{13}^{r_{1}-1}-x_{3}^{r_{1}-1}\right) x_{3}^{r_{2}-1} \zeta\left(r_{1}, r_{2}, 1\right) & +\sum_{r_{1} \geq 2} x_{1}^{r_{1}-1} \zeta\left(r_{1}, 1,1\right),
\end{aligned}
$$


which is just (3.2) under the operation $x_{1} \leftrightarrow x_{3}$. Further,

$$
\begin{gathered}
(3.6)=G_{3}\left(x_{123}, x_{23}, x_{3}\right)-G_{3}\left(x_{23}, x_{23}, x_{2}\right)-G_{3}\left(x_{13}, x_{3}, x_{3}\right) \\
+G_{3}\left(x_{3}, x_{3}, x_{3}\right)-\sum_{r_{1} \geq 2, r_{2} \geq 1}\left(x_{12}^{r_{1}-1}-x_{2}^{r_{1}-1}\right) x_{2}^{r_{2}-1} \zeta\left(r_{1}, r_{2}, 1\right) \\
+\sum_{r_{1} \geq 2} x_{1}^{r_{1}-1} \zeta\left(r_{1}, 1,1\right),
\end{gathered}
$$

which is (3.2) under the operation Cyc $\left(x_{1} \rightarrow x_{2} \rightarrow x_{3} \rightarrow x_{1}\right)$. By the same argument, we easily find that (3.3), (3.5) and (3.7) can all be obtained from (3.2) under different permutations of $x_{1}, x_{2}$ and $x_{3}$. Therefore,

$$
\begin{aligned}
& \sum_{s_{1}, s_{2}, s_{3} \geq 2} x_{1}^{s_{1}-1} x_{2}^{s_{2}-1} x_{3}^{s_{3}-1} \zeta\left(s_{1}\right) \zeta\left(s_{2}\right) \zeta\left(s_{3}\right) \\
&= \bigoplus_{\substack{\mathcal{S}\left(x_{1}, x_{2}, x_{3}\right)\\
}}\left\{G_{3}\left(x_{2}, x_{2}, x_{2}\right)+G_{3}\left(x_{123}, x_{12}, x_{2}\right)\right. \\
&-\sum_{\substack{t_{1}, s_{3}, r_{1} \geq 2, r_{2} \geq 1 \\
r_{1}+r_{2}=t_{1}+s_{3}}} x_{1}^{r_{2}-1}\left(x_{13}^{r_{1}-1}-x_{12}, x_{2}\right)-G_{3}\left(x_{23}, x_{2}, x_{2}\right) \\
& \\
&\left.+\sum_{r_{1} \geq 2}^{r_{1}-1}\right) \zeta\left(r_{1}, r_{2}, 1\right)
\end{aligned}
$$

where, for a function $f\left(x_{1}, \ldots, x_{k}\right)$, we define

$$
\bigoplus_{\mathcal{S}\left(x_{1}, \ldots, x_{k}\right)} f\left(x_{1}, \ldots, x_{k}\right)=\sum_{\sigma: \text { permutations of } 1, \ldots, k} f\left(x_{\sigma(1)}, \ldots, x_{\sigma(k)}\right) .
$$

3.2. Second method. Multiplying $\zeta\left(s_{3}\right)$ on $(1.2)$ and using stuffle relations, we get

$$
\begin{aligned}
& \sum_{s_{1}, s_{2}, s_{3} \geq 2} x_{1}^{s_{1}-1} x_{2}^{s_{2}-1} x_{3}^{s_{3}-1} \zeta\left(s_{1}\right) \zeta\left(s_{2}\right) \zeta\left(s_{3}\right) \\
= & \sum_{s_{1}, s_{2}, s_{3} \geq 2} x_{1}^{s_{1}-1} x_{2}^{s_{2}-1} x_{3}^{s_{3}-1}
\end{aligned}
$$




$$
\begin{aligned}
& \cdot \sum_{\substack{t_{1} \geq 2, t_{2} \geq 1 \\
t_{1}+t_{2}=s_{1}+s_{2}}}\left\{\left[\begin{array}{l}
t_{1} \\
s_{1}
\end{array}\right]+\left[\begin{array}{l}
t_{1} \\
s_{2}
\end{array}\right]\right\} \zeta\left(t_{1}, t_{2}, s_{3}\right) \\
& +\sum_{s_{1}, s_{2}, s_{3} \geq 2} x_{1}^{s_{1}-1} x_{2}^{s_{2}-1} x_{3}^{s_{3}-1} \\
& \cdot \sum_{\substack{t_{1} \geq 2, t_{2} \geq 1 \\
t_{1}+t_{2}=s_{1}+s_{2}}}\left\{\left[\begin{array}{l}
t_{1} \\
s_{1}
\end{array}\right]+\left[\begin{array}{l}
t_{1} \\
s_{2}
\end{array}\right]\right\} \zeta\left(t_{1}, s_{3}, t_{2}\right) \\
& +\sum_{s_{1}, s_{2}, s_{3} \geq 2} x_{1}^{s_{1}-1} x_{2}^{s_{2}-1} x_{3}^{s_{3}-1} \\
& \sum_{\substack{t_{1} \geq 2, t_{2} \geq 1 \\
t_{1}+t_{2}=s_{1}+s_{2}}}\left\{\left[\begin{array}{l}
t_{1} \\
s_{1}
\end{array}\right]+\left[\begin{array}{l}
t_{1} \\
s_{2}
\end{array}\right]\right\} \zeta\left(s_{3}, t_{1}, t_{2}\right) \\
& +\sum_{s_{1}, s_{2}, s_{3} \geq 2} x_{1}^{s_{1}-1} x_{2}^{s_{2}-1} x_{3}^{s_{3}-1} \\
& \sum_{\substack{t_{1} \geq 2, t_{2} \geq 1 \\
t_{1}+t_{2}=s_{1}+s_{2}}}\left\{\left[\begin{array}{l}
t_{1} \\
s_{1}
\end{array}\right]+\left[\begin{array}{l}
t_{1} \\
s_{2}
\end{array}\right]\right\} \zeta\left(t_{1}, t_{2}+s_{3}\right) \\
& +\sum_{s_{1}, s_{2}, s_{3} \geq 2} x_{1}^{s_{1}-1} x_{2}^{s_{2}-1} x_{3}^{s_{3}-1} \\
& \cdot \sum_{\substack{t_{1} \geq 2, t_{2} \geq 1 \\
t_{1}+t_{2}=s_{1}+s_{2}}}\left\{\left[\begin{array}{l}
t_{1} \\
s_{1}
\end{array}\right]+\left[\begin{array}{l}
t_{1} \\
s_{2}
\end{array}\right]\right\} \zeta\left(t_{1}+s_{3}, t_{2}\right) \text {. }
\end{aligned}
$$

Using techniques similar to the one used in the preceding subsection, we get

$$
\begin{aligned}
(3.8)= & \bigoplus_{\mathcal{S}\left(x_{1}, x_{2}\right)}\left\{G_{3}\left(x_{12}, x_{2}, x_{3}\right)-G_{3}\left(x_{2}, x_{2}, x_{3}\right)\right. \\
& -\sum_{t_{1} \geq 2, s_{3} \geq 1} x_{1}^{t_{1}-1} x_{3}^{s_{3}-1} \zeta\left(t_{1}, 1, s_{3}\right) \\
& +\sum_{t_{1} \geq 2} x_{1}^{t_{1}-1} \zeta\left(t_{1}, 1,1\right) \\
& -\sum_{t_{1} \geq 2, t_{2} \geq 1} x_{12}^{t_{1}-1} x_{2}^{t_{2}-1} \zeta\left(t_{1}, t_{2}, 1\right)
\end{aligned}
$$




$$
\begin{aligned}
& \left.+\sum_{t_{1} \geq 2, t_{2} \geq 1} x_{2}^{t_{1}+t_{2}-2} \zeta\left(t_{1}, t_{2}, 1\right)\right\} \\
& (3.9)=\bigoplus_{\mathcal{S}\left(x_{1}, x_{2}\right)}\left\{G_{3}\left(x_{12}, x_{3}, x_{2}\right)-G_{3}\left(x_{2}, x_{3}, x_{2}\right)\right. \\
& -\sum_{t_{1} \geq 2, s_{3} \geq 1} x_{1}^{t_{1}-1} x_{3}^{s_{3}-1} \zeta\left(t_{1}, s_{3}, 1\right) \\
& +\sum_{t_{1} \geq 2} x_{1}^{t_{1}-1} \zeta\left(t_{1}, 1,1\right) \\
& -\sum_{t_{1} \geq 2, t_{2} \geq 1} x_{12}^{t_{1}-1} x_{2}^{t_{2}-1} \zeta\left(t_{1}, 1, t_{2}\right) \\
& \left.+\sum_{t_{1} \geq 2, t_{2} \geq 1} x_{2}^{t_{1}+t_{2}-2} \zeta\left(t_{1}, 1, t_{2}\right)\right\} \\
& (3.10)=\bigoplus_{\mathcal{S}\left(x_{1}, x_{2}\right)}\left\{G_{3}\left(x_{3}, x_{12}, x_{2}\right)-G_{3}\left(x_{3}, x_{2}, x_{2}\right)\right. \\
& -\sum_{s_{3} \geq 2, t_{1} \geq 1} x_{1}^{t_{1}-1} x_{3}^{s_{3}-1} \zeta\left(s_{3}, t_{1}, 1\right) \\
& \left.+\sum_{s_{3} \geq 2} x_{3}^{s_{3}-1} \zeta\left(s_{3}, 1,1\right)\right\} \\
& (3.11)=\bigoplus_{\mathcal{S}\left(x_{1}, x_{2}\right)}\left\{\frac{G_{2}\left(x_{12}, x_{3}\right)-G_{2}\left(x_{12}, x_{2}\right)}{x_{3}-x_{2}}-\frac{G_{2}\left(x_{12}, x_{2}\right)}{x_{2}}\right. \\
& -\frac{G_{2}\left(x_{1}, x_{3}\right)}{x_{3}}-\frac{G_{2}\left(x_{2}, x_{3}\right)-G_{2}\left(x_{2}, x_{2}\right)}{x_{3}-x_{2}} \\
& +\frac{1}{x_{2}} \sum_{t_{1} \geq 2}\left(x_{12}^{t_{1}-1}-x_{2}^{t_{1}-1}\right) \zeta\left(t_{1}, 1\right) \\
& \left.+\frac{G_{2}\left(x_{2}, x_{2}\right)}{x_{2}}+\frac{1}{x_{3}} \sum_{t_{1} \geq 1} x_{1}^{t_{1}-1}\left(\zeta\left(t_{1}, 1\right)+x_{3} \zeta\left(t_{1}, 2\right)\right)\right\} \\
& (3.12)=\bigoplus_{\mathcal{S}\left(x_{1}, x_{2}\right)}\left\{\frac{G_{2}\left(x_{3}, x_{2}\right)-G_{2}\left(x_{12}, x_{2}\right)}{x_{3}-x_{12}}\right. \\
& +\frac{G_{2}\left(x_{2}, x_{2}\right)}{x_{2}}-\frac{G_{2}\left(x_{3}, x_{2}\right)-G_{2}\left(x_{2}, x_{2}\right)}{x_{3}-x_{2}}
\end{aligned}
$$




$$
\begin{aligned}
& -\frac{G_{2}\left(x_{12}, x_{2}\right)}{x_{12}} \\
& \left.-\sum_{t \geq 2}\left(\frac{x_{3}^{t-1}-x_{1}^{t-1}}{x_{3}-x_{1}}-x_{3}^{t-2}-x_{1}^{t-2}\right) \zeta(t, 1)-\zeta(2,1)\right\} .
\end{aligned}
$$

Therefore,

$$
\begin{aligned}
& \sum_{s_{1}, s_{2}, s_{3} \geq 2} x_{1}^{s_{1}-1} x_{2}^{s_{2}-1} x_{3}^{s_{3}-1} \zeta\left(s_{1}\right) \zeta\left(s_{2}\right) \zeta\left(s_{3}\right) \\
= & \bigoplus_{\mathcal{S}\left(x_{1}, x_{2}\right)}\left\{G_{3}\left(x_{12}, x_{2}, x_{3}\right)+G_{3}\left(x_{12}, x_{3}, x_{2}\right)-G_{3}\left(x_{2}, x_{3}, x_{2}\right)\right. \\
& +G_{3}\left(x_{3}, x_{12}, x_{2}\right)-G_{3}\left(x_{3}, x_{2}, x_{2}\right)-G_{3}\left(x_{2}, x_{2}, x_{3}\right) \\
& +\frac{G_{2}\left(x_{12}, x_{3}\right)-G_{2}\left(x_{12}, x_{2}\right)}{x_{3}-x_{2}}-\frac{G_{2}\left(x_{12}, x_{2}\right)}{x_{2}} \\
& -\frac{G_{2}\left(x_{2}, x_{3}\right)-G_{2}\left(x_{2}, x_{2}\right)}{x_{3}-x_{2}}+\frac{2}{x_{2}} G_{2}\left(x_{2}, x_{2}\right)-\frac{G_{2}\left(x_{1}, x_{3}\right)}{x_{3}} \\
& +\frac{G_{2}\left(x_{3}, x_{2}\right)-G_{2}\left(x_{12}, x_{2}\right)}{x_{3}-x_{2}-x_{1}}-\frac{G_{2}\left(x_{12}, x_{2}\right)}{x_{12}} \\
& -\sum_{t_{1} \geq 2, t_{2} \geq 1} x_{12}^{t_{1}-1} x_{2}^{t_{2}-1} \zeta\left(t_{1}, t_{2}, 1\right)-\sum_{t_{1} \geq 2, t_{2} \geq 1} x_{3}^{t_{1}-1} x_{1}^{t_{2}-1} \zeta\left(t_{1}, t_{2}, 1\right) \\
& +\sum_{t_{1} \geq 2, t_{2} \geq 1} x_{2}^{t_{1}+t_{2}-2} \zeta\left(t_{1}, t_{2}, 1\right) \\
& +\sum_{t_{1} \geq 2, t_{2} \geq 1} x_{2}^{t_{1}+t_{2}-2} \zeta\left(t_{1}, 1, t_{2}\right) \\
& -\sum_{t_{1} \geq 2, t_{2} \geq 1} x_{1}^{t_{1}-1} x_{3}^{t_{2}-1} \zeta\left(t_{1}, t_{2}, 1\right)-\sum_{t_{1} \geq 2, t_{2} \geq 1} x_{12}^{t_{1}-1} x_{2}^{t_{2}-1} \zeta\left(t_{1}, 1, t_{2}\right) \\
& +\frac{1}{x_{2}} \sum_{t_{1} \geq 2} x_{12}^{t_{1}-1} \zeta\left(t_{1}, 1\right)+2 \sum_{t_{1} \geq 2} x_{1}^{t_{1}-1} \zeta\left(t_{1}, 1,1\right) \\
& +\sum_{t_{1} \geq 2} x_{3}^{t_{1}-1} \zeta\left(t_{1}, 1,1\right)-\sum_{t_{1} \geq 2, t_{2} \geq 1} x_{1}^{t_{1}-1} x_{3}^{t_{2}-1} \zeta\left(t_{1}, 1, t_{2}\right) \\
& +\frac{1}{x_{3}} \sum_{t_{1} \geq 2} x_{1}^{t_{1}-1}\left(\zeta\left(t_{1}, 1\right)+x_{3} \zeta\left(t_{1}, 2\right)\right) \\
&
\end{aligned}
$$




$$
\begin{aligned}
& -\frac{G_{2}\left(x_{3}, x_{2}\right)-G_{2}\left(x_{2}, x_{2}\right)}{x_{3}-x_{2}}-\sum_{t \geq 2}\left(\frac{x_{3}^{t-1}-x_{1}^{t-1}}{x_{3}-x_{1}}-x_{3}^{t-2}\right) \zeta(t, 1) \\
& -\zeta(2,1)\} .
\end{aligned}
$$

3.3. Third method. Equation (1.1) yields, with repeated use of stuffle relations,

$$
\begin{gathered}
\zeta\left(s_{1}\right) \zeta\left(s_{2}\right) \zeta\left(s_{3}\right)=\zeta\left(s_{1}, s_{2}, s_{3}\right)+\zeta\left(s_{1}, s_{3}, s_{2}\right)+\zeta\left(s_{3}, s_{1}, s_{2}\right) \\
+\zeta\left(s_{1}, s_{2}+s_{3}\right)+\zeta\left(s_{1}+s_{3}, s_{2}\right)+\zeta\left(s_{2}, s_{1}, s_{3}\right) \\
+\zeta\left(s_{2}, s_{3}, s_{1}\right)+\zeta\left(s_{3}, s_{2}, s_{1}\right)+\zeta\left(s_{2}, s_{1}+s_{3}\right) \\
+\zeta\left(s_{2}+s_{3}, s_{1}\right)+\zeta\left(s_{1}+s_{2}, s_{3}\right)+\zeta\left(s_{3}, s_{1}+s_{2}\right) \\
+\zeta\left(s_{1}+s_{2}+s_{3}\right)
\end{gathered}
$$

On the right hand side of the above there are essentially four types of MZVs. Similar computations as above lead to the following four expressions of their generating functions: (write $\mathbf{x}^{\mathbf{s}-\mathbf{1}}=x_{1}^{s_{1}-1} x_{2}^{s_{2}-1} x_{3}^{s_{3}-1}$ )

$$
\begin{aligned}
& \sum_{s_{1}, s_{2}, s_{3} \geq 2} \mathbf{x}^{\mathbf{s}-\mathbf{1}} \zeta\left(s_{1}, s_{2}, s_{3}\right)=G_{3}\left(x_{1}, x_{2}, x_{3}\right) \\
& +\sum_{s_{1} \geq 2} x_{1}^{s_{1}-1} \zeta\left(s_{1}, 1,1\right)-\sum_{s_{1} \geq 2, s_{3} \geq 1} x_{1}^{s_{1}-1} x_{3}^{s_{3}-1} \zeta\left(s_{1}, 1, s_{3}\right) \\
& -\sum_{s_{1} \geq 2, s_{2} \geq 1} x_{1}^{s_{1}-1} x_{2}^{s_{2}-1} \zeta\left(s_{1}, s_{2}, 1\right), \sum_{s_{1}, s_{2}, s_{3} \geq 2} \mathbf{x}^{\mathbf{s}-\mathbf{1}} \zeta\left(s_{1}, s_{2}+s_{3}\right) \\
= & \frac{G_{2}\left(x_{1}, x_{3}\right)-G_{2}\left(x_{1}, x_{2}\right)}{x_{3}-x_{2}}-\frac{G_{2}\left(x_{1}, x_{2}\right)}{x_{2}} \\
& -\frac{G_{2}\left(x_{1}, x_{3}\right)}{x_{3}}+\sum_{s_{1} \geq 2} x_{1}^{s_{1}-1} \zeta\left(s_{1}, 2\right) \\
& +\left(\frac{1}{x_{3}}+\frac{1}{x_{2}}\right) \sum_{s_{1} \geq 2} x_{1}^{s_{1}-1} \zeta\left(s_{1}, 1\right), \\
& \sum_{s_{1}, s_{2}, s_{3} \geq 2} \mathbf{x}^{\mathbf{s}-\mathbf{1}} \zeta\left(s_{2}+s_{3}, s_{1}\right) \\
= & \frac{G_{2}\left(x_{3}, x_{1}\right)-G_{2}\left(x_{2}, x_{1}\right)}{x_{3}-x_{2}}-\frac{G_{2}\left(x_{2}, x_{1}\right)}{x_{2}}-\frac{G_{2}\left(x_{3}, x_{1}\right)}{x_{3}}
\end{aligned}
$$




$$
\begin{aligned}
& +\sum_{s_{1} \geq 1} x_{1}^{s_{1}-1} \zeta\left(2, s_{1}\right) \\
& -\sum_{s \geq 2}\left(\frac{x_{3}^{s-1}-x_{2}^{s-1}}{x_{3}-x_{2}}-x_{3}^{s-2}-x_{2}^{s-2}+\delta_{s, 2}\right) \zeta(s, 1), \\
& \sum_{s_{1}, s_{2}, s_{3} \geq 2} \mathbf{x}^{\mathbf{s}-1} \zeta\left(s_{1}+s_{2}+s_{3}\right) \\
= & \frac{G_{1}\left(x_{1}\right)}{x_{1}^{2}}-\zeta(3)-\frac{\zeta(2)}{x_{1}}-\frac{\zeta(2)}{x_{3}} \\
+ & \frac{1}{x_{3}-x_{2}}\left(\frac{G_{1}\left(x_{3}\right)-G_{1}\left(x_{1}\right)}{x_{3}-x_{1}}-\frac{G_{1}\left(x_{3}\right)}{x_{3}}\right. \\
& \left.-\frac{G_{1}\left(x_{2}\right)-G_{1}\left(x_{1}\right)}{x_{2}-x_{1}}+\frac{G_{1}\left(x_{2}\right)}{x_{2}}\right) \\
& -\frac{1}{x_{2}}\left(\frac{G_{1}\left(x_{2}\right)-G_{1}\left(x_{1}\right)}{x_{2}-x_{1}}-\frac{G_{1}\left(x_{2}\right)}{x_{2}}-\frac{G_{1}\left(x_{1}\right)}{x_{1}}+\zeta(2)\right) \\
+ & \frac{1}{x_{3}}\left(\frac{G_{1}\left(x_{3}\right)}{\left.x_{3}+\frac{G_{1}\left(x_{1}\right)}{x_{1}}-\frac{G_{1}\left(x_{3}\right)-G_{1}\left(x_{1}\right)}{x_{3}-x_{1}}\right) .}\right.
\end{aligned}
$$

Therefore, (3.13) becomes

$$
\begin{aligned}
& \sum_{s_{1}, s_{2}, s_{3} \geq 2} \mathbf{x}^{\mathbf{s}-\mathbf{1}} \zeta\left(s_{1}\right) \zeta\left(s_{2}\right) \zeta\left(s_{3}\right) \\
= & \bigoplus_{\mathcal{S}\left(x_{1}, x_{2}, x_{3}\right)}\left\{G_{3}\left(x_{1}, x_{2}, x_{3}\right)-\frac{G_{2}\left(x_{1}, x_{2}\right)}{x_{2}}\right. \\
- & \frac{G_{2}\left(x_{2}, x_{1}\right)}{x_{2}}+\frac{G_{2}\left(x_{1}, x_{3}\right)+G_{2}\left(x_{3}, x_{1}\right)}{x_{3}-x_{2}} \\
- & \sum_{s_{1} \geq 2, s_{2} \geq 1} x_{1}^{s_{1}-1} x_{2}^{s_{2}-1} \zeta\left(s_{1}, s_{2}, 1\right) \\
+ & \sum_{s \geq 2} x_{1}^{s-2} \zeta(s, 1)+\sum_{s \geq 2} x_{1}^{s-1} \zeta(s, 1,1) \\
- & \sum_{s_{1} \geq 2, s_{3} \geq 1} x_{1}^{s_{1}-1} x_{3}^{s_{3}-1} \zeta\left(s_{1}, 1, s_{3}\right) \\
+ & \left.\frac{1}{x_{2}} \sum_{s \geq 2} x_{1}^{s-1} \zeta(s, 1)\right\}-\zeta(3)
\end{aligned}
$$




$$
\begin{aligned}
& +\bigoplus_{\mathcal{C}\left(x_{1}, x_{2}, x_{3}\right)}\left\{\sum_{s \geq 2} x_{1}^{s-1} \zeta(s, 2)\right. \\
& +\sum_{s \geq 2} x_{1}^{s-1} \zeta(2, s)-\sum_{s \geq 2}\left(\frac{x_{3}^{s-1}-x_{2}^{s-1}}{x_{3}-x_{2}}\right) \zeta(s, 1) \\
& \left.+\frac{G_{1}\left(x_{1}\right)}{x_{1}^{2}}-\frac{\zeta(2)}{x_{1}}\right\} \\
& +\bigoplus_{\mathcal{C}\left(x_{2}, x_{3}\right)}\left\{\frac{1}{x_{3}-x_{2}}\left(\frac{G_{1}\left(x_{3}\right)-G_{1}\left(x_{1}\right)}{x_{3}-x_{1}}+\frac{G_{1}\left(x_{2}\right)}{x_{2}}\right)\right. \\
& \left.-\frac{1}{x_{2}}\left(\frac{G_{1}\left(x_{2}\right)-G_{1}\left(x_{1}\right)}{x_{2}-x_{1}}-\frac{G_{1}\left(x_{1}\right)}{x_{1}}\right)\right\} .
\end{aligned}
$$

Here, for a function $f\left(x_{1}, \ldots, x_{k}\right)$, we define

$$
\bigoplus_{\mathcal{C}\left(x_{1}, \ldots, x_{k}\right)} f\left(x_{1}, \ldots, x_{k}\right)=\sum_{i=1}^{k} f\left(x_{i}, x_{i+1}, \ldots, x_{i+k-1}\right),
$$

where the subscript is taken modulo $k$.

By comparing the first and the third methods, we get:

Theorem 3.1. We have

$$
\begin{aligned}
& \bigoplus_{\mathcal{S}\left(x_{1}, x_{2}, x_{3}\right)}\left\{G_{3}\left(x_{123}, x_{12}, x_{2}\right)-G_{3}\left(x_{12}, x_{12}, x_{2}\right)\right. \\
& -G_{3}\left(x_{23}, x_{2}, x_{2}\right)+G_{3}\left(x_{2}, x_{2}, x_{2}\right)-G_{3}\left(x_{1}, x_{2}, x_{3}\right) \\
& \left.-\frac{G_{2}\left(x_{1}, x_{3}\right)+G_{2}\left(x_{3}, x_{1}\right)}{x_{3}-x_{2}}+\frac{G_{2}\left(x_{1}, x_{2}\right)}{x_{2}}+\frac{G_{2}\left(x_{2}, x_{1}\right)}{x_{2}}\right\} \\
& -\bigoplus_{\mathcal{C}\left(x_{2}, x_{3}\right)}\left\{\frac{1}{x_{3}-x_{2}}\left(\frac{G_{1}\left(x_{3}\right)-G_{1}\left(x_{1}\right)}{x_{3}-x_{1}}+\frac{G_{1}\left(x_{2}\right)}{x_{2}}\right)\right. \\
& \left.-\frac{G_{1}\left(x_{2}\right)-G_{1}\left(x_{1}\right)}{x_{2}\left(x_{2}-x_{1}\right)}+\frac{G_{1}\left(x_{1}\right)}{x_{1} x_{2}}\right\} \\
= & \bigoplus_{\mathcal{S}\left(x_{1}, x_{2}, x_{3}\right)}\left\{\sum_{r_{1} \geq 2, r_{2} \geq 1} x_{1}^{r_{2}-1}\left(x_{13}^{r_{1}-1}-x_{1}^{r_{1}-1}-x_{3}^{r_{1}-1}\right)\right. \\
& \cdot \zeta\left(r_{1}, r_{2}, 1\right)+\sum_{s \geq 2} x_{1}^{s-2} \zeta(s, 1)
\end{aligned}
$$




$$
\begin{aligned}
& -\sum_{s_{1} \geq 2, s_{3} \geq 1} x_{1}^{s_{1}-1} x_{3}^{s_{3}-1} \zeta\left(s_{1}, 1, s_{3}\right) \\
& \left.+\frac{1}{x_{2}} \sum_{s \geq 2} x_{1}^{s-1} \zeta(s, 1)-\sum_{s \geq 2}\left(\frac{x_{3}^{s-1}}{x_{3}-x_{2}}\right) \zeta(s, 1)\right\} \\
& +\bigoplus_{\mathcal{C}\left(x_{1}, x_{2}, x_{3}\right)}\left\{\sum_{s \geq 2} x_{1}^{s-1} \zeta(s, 2)+\sum_{s \geq 2} x_{1}^{s-1} \zeta(2, s)\right. \\
& \left.+\frac{G_{1}\left(x_{1}\right)}{x_{1}^{2}}-\frac{\zeta(2)}{x_{1}}\right\}-\zeta(3) .
\end{aligned}
$$

Proof. Clear.

This theorem is equivalent to the following result, which can be regarded as a parametric family of weighted sum formulas.

Theorem 3.2. Let $a, b$ and $c$ be any real numbers, and let $\sigma=a+b$. Then for any positive integer $n \geq 4$, we have

$$
\begin{aligned}
\bigoplus_{\mathcal{S}(a, b, c)}\left\{\sum _ { \substack { j \geq 2 , k , l \geq 1 \\
j + k + l = n } } \left(a c(a+b+c)^{j-1}(a+b)^{k-1} b^{l}\right.\right. \\
-a c(a+b)^{j+k-2} b^{l}-a c(b+c)^{j-1} b^{k+l-1} \\
\left.+a c b^{j+k+l-2}-a^{j} b^{k} c^{l}\right) \zeta(j, k, l) \\
\left.+\sum_{j \geq 2, k \geq 1}\left(c a^{j} b^{k-1}+c a^{k} b^{j-1}-\frac{b\left(a^{j} c^{k}+a^{k} c^{j}\right)}{c-b}\right) \zeta(j, k)\right\} \\
-\bigoplus_{\mathcal{C}(b, c)}\left\{\frac{1}{c-b}\left(\frac{a b c^{n}-b c a^{n}}{c-a}+a c b^{n-1}\right)\right. \\
\left.-\frac{a c\left(b^{n-1}-a^{n-1}\right)}{b-a}+c a^{n-1}\right\} \zeta(n) \\
=\bigoplus_{\mathcal{S}(a, b, c)}\left\{\sum _ { \substack { j \geq 2 , k \geq 1 \\
j + k = n - 1 } } \left(a ^ { k } b c \left[(a+c)^{j-1}\right.\right.\right. \\
\left.\left.\quad-a^{j-1}-c^{j-1}\right] \zeta(j, k, 1)-a^{j} b c^{k} \zeta(j, 1, k)\right)
\end{aligned}
$$




$$
\begin{aligned}
& +\left(a^{n-1} c+a^{n-2} b c-\frac{a b c^{n-1}}{c-b}\right) \zeta(n-1,1) \\
& \left.+\frac{1}{2} a^{n-2} b c(\zeta(n-2,2)+\zeta(2, n-2))+\frac{1}{2} a^{n-2} b c \zeta(n)\right\} .
\end{aligned}
$$

Proof. In Theorem 3.1, we first set $x_{1}=a t, x_{2}=b t$ and $x_{3}=c t$. Then, by comparing the coefficient of $t^{n-3}$, we get Theorem 3.2.

The following weighted sum formula seems to be new.

Corollary 3.3. For any positive integer $n \geq 2$, we have

$$
\begin{aligned}
\sum_{\substack{j \geq 2, k, l \geq 1 \\
j+k+l=n}} 2^{j-1} \zeta(j, k, l)+\sum_{\substack{j \geq 2, k \geq 1 \\
j+k=n-1}} 2^{j} \zeta(j, k, 1) \\
=n \zeta(n-1,1)+3 \zeta(n-2,2)+\zeta(2, n-2)+2 \zeta(n) .
\end{aligned}
$$

Proof. Setting $a=1$ and letting $b \rightarrow 1$ and then $c \rightarrow 1$ in Theorem 3.2, we get

$$
\begin{aligned}
& \sum_{\substack{j \geq 2, k, l \geq 1 \\
j+k+l=n}} 6\left(3^{j-1} 2^{k-1}-2^{j+k-2}-2^{j-1}\right) \zeta(j, k, l) \\
& +\sum_{\substack{j \geq 2, k \geq 1 \\
j+k=n}} 3(6-n) \zeta(j, k)-\frac{n^{2}-9 n+20}{2} \zeta(n) \\
= & \sum_{\substack{j \geq 2, k \geq 1 \\
j+k=n-1}} 6\left(\left(2^{j-1}-2\right) \zeta(j, k, 1)-\zeta(j, 1, k)\right) \\
& +3(6-n) \zeta(n-1,1)+3 \zeta(n-2,2)+3 \zeta(2, n-2) .
\end{aligned}
$$

The following two special type sum formulas are both special cases of [6, Theorem 5.1]:

$$
\begin{aligned}
& \sum_{\substack{j \geq 2, k \geq 1 \\
j+k=n-1}} \zeta(j, 1, k)=\zeta(2, n-1)+\zeta(n, 1), \\
& \sum_{\substack{j \geq 2, k \geq 1 \\
j+k=n-1}} \zeta(j, k, 1)=\zeta(n-1,1)+\zeta(n-2,2) .
\end{aligned}
$$


Notice that (3.15) is also a special case of Eie's generalized sum formula in [1]. Combining with sum formula (2.1), we get

$$
\begin{aligned}
& \sum_{\substack{j \geq 2, k, l \geq 1 \\
j+k+l=n}} 6\left(3^{j-1} 2^{k-1}-2^{j+k-2}-2^{j-1}\right) \zeta(j, k, l) \\
= & \frac{n^{2}-3 n-16}{2} \zeta(n)+3 \sum_{\substack{j \geq 2, k \geq 1 \\
j+k=n-1}} 2^{j} \zeta(j, k, 1) \\
& -3 n \zeta(n-1,1)-9 \zeta(n-2,2)-3 \zeta(2, n-2) .
\end{aligned}
$$

Dividing by 3 throughout, we get

$$
\begin{aligned}
& \sum_{\substack{j \geq 2, k, l \geq 1 \\
j+k+l=n}}\left(3^{j-1} 2^{k}-2^{j+k-1}-2^{j}\right) \zeta(j, k, l)-\frac{n^{2}-3 n-16}{6} \zeta(n) \\
= & \sum_{\substack{j \geq 2, k \geq 1 \\
j+k=n-1}} 2^{j} \zeta(j, k, 1)-n \zeta(n-1,1) \\
& -3 \zeta(n-2,2)-\zeta(2, n-2) .
\end{aligned}
$$

Hence, the corollary follows from [9, Corollary 4.1].

By comparing the second and the third methods, we can get another identity involving the generating function $G_{3}$. However, it is quite long, so here we just write down the following version concerning a parametric family of weighted sum formulas.

Theorem 3.4. Let $a, b$ and $c$ be any real numbers, and let $\sigma=a+b$. Then, for any positive integer $n \geq 2$, we have:

$$
\begin{aligned}
& \sum_{\substack{j \geq 2, k, l \geq 1 \\
j+k+l=n}} \bigoplus_{\mathcal{C}(a, b)}\left(a \sigma^{j-1}\left(b^{k} c^{l}+b^{l} c^{k}\right)+a \sigma^{k-1} b^{l} c^{j}\right. \\
& \left.-\bigoplus_{\mathcal{C}(j, k, l)}\left(a^{j+k-1} b c^{l}+a^{j} b^{k} c^{l}\right)\right) \zeta(j, k, l) \\
& -\sum_{\substack{j \geq 2, k \geq 1 \\
j+k=n-1}} \bigoplus_{\mathcal{C}(a, b)}\left(a \sigma^{j-1} b^{k} c-a^{j+k-1} b c-a^{j} b^{k} c\right) \zeta(j, k, 1)
\end{aligned}
$$




$$
\begin{aligned}
& -\sum_{\substack{j \geq 2, k \geq 1 \\
j+k=n-1}} \bigoplus_{\mathcal{C}(a, b)}\left(a \sigma^{j-1} b^{k} c-a^{j+k-1} b c-a^{k} b c^{j}-a^{k} c b^{j}\right) \zeta(j, 1, k) \\
& =\sum_{\substack{j \geq 2, k \geq 1 \\
j+k=n}}\left(\bigoplus_{\mathcal{C}(a, b, c)} \bigoplus_{\mathcal{C}(j, k)}\left(\frac{a^{j}\left(b c^{k}-c b^{k}\right)}{c-b}-a^{j} b^{k-1} c-a^{j} b c^{k-1}\right)\right. \\
& -\bigoplus_{\mathcal{C}(a, b)}\left(\frac{\sigma^{j-1} a\left(b c^{k}-c b^{k}\right)}{c-b}-a^{j} b c^{k-1}\right. \\
& -\sigma^{j-1} b^{k-1} a c+\frac{a b^{k} c^{j}-\sigma^{j-1} a c b^{k}}{c-b-a}-\sigma^{j-2} b^{k} a c \\
& \left.\left.+\bigoplus_{\mathcal{C}(j, k)}\left(a c b^{j+k-2}-\frac{b^{k-1} a\left(b c^{j}-c b^{j}\right)}{c-b}\right)\right)\right) \zeta(j, k) \\
& -\bigoplus_{\mathcal{C}(a, b)}\left(a c \sigma^{n-2}-a^{n-1} c-c^{n-1} a-2 a b^{n-2} c\right. \\
& \left.+\frac{a c b^{n-1}-b c a^{n-1}}{2(b-a)}\right) \zeta(n-1,1) \\
& +\bigoplus_{\mathcal{C}(b, c)}\left(\frac{b^{2}\left(a^{2} c^{n-1}-a^{n} c\right)}{c(c-b)(c-a)}+a^{n-1} b\right) \zeta(n) \\
& +b c a^{n-2} \zeta(n)+a b c^{n-2} \zeta(n-2,2) \\
& +\bigoplus_{\mathcal{C}(a, b, c)} a^{n-2} b c \zeta(2, n-2)-\delta_{n, 2}(a b+a c+b c) \zeta(2) \\
& -\delta_{n, 3} a b c(\zeta(2,1)+\zeta(3)) \text {. }
\end{aligned}
$$

Proof. Similar to that of Theorem 3.2. We leave the details to the interested reader.

Corollary 3.5. Let $n$ be any positive integer. Then

$$
\begin{aligned}
& \sum_{\substack{j \geq 2, k, l \geq 1 \\
j+k+l=n}}\left(2^{j+1}+2^{k}\right) \zeta(j, k, l) \\
& -\sum_{\substack{j \geq 2, k \geq 1 \\
j+k=n-1}} 2^{j}(\zeta(j, 1, k)+\zeta(j, k, 1))+\sum_{\substack{j \geq 2, k \geq 1 \\
j+k=n}} 2^{j} \cdot k \cdot \zeta(j, k)
\end{aligned}
$$




$$
\begin{aligned}
= & \frac{(n+3)(n+1)}{2} \zeta(n)-3 \zeta(n-2,2) \\
& -\left(2^{n-1}+n\right) \zeta(n-1,1)-3 \zeta(2, n-2) .
\end{aligned}
$$

Proof. Setting $a=1$ and letting $b, c \rightarrow 1$ in Theorem 3.4, we get

$$
\begin{aligned}
& \sum_{\substack{j \geq 2, k, l \geq 1 \\
j+k+l=n}}\left(2^{j+1}+2^{k}-6\right) \zeta(j, k, l) \\
& -\sum_{\substack{j \geq 2, k \geq 1 \\
j+k=n-1}}\left[\left(2^{j}-6\right) \zeta(j, 1, k)+\left(2^{j}-4\right) \zeta(j, k, 1)\right] \\
& +\sum_{\substack{j \geq 2, k \geq 1 \\
j+k=n}}\left(2^{j}(k-1)-2^{j-1}-5 n+22\right) \zeta(j, k) \\
= & \frac{n^{2}-9 n+32}{2} \zeta(n)+\zeta(n-2,2) \\
& -\left(2^{n-1}+n-10\right) \zeta(n-1,1)+3 \zeta(2, n-2) .
\end{aligned}
$$

Using the weighted sum formula (1.6), the sum formulas (2.2), (3.14) and (3.15), we can derive our corollary quickly.

4. Depth 3: product of zeta and double zeta. Set $x^{s-1}=$ $x_{1}^{s_{1}-1} x_{2}^{s_{2}-1} x_{3}^{s_{3}-1}$ throughout this section. By (1.3) for all $s_{1}, s_{3} \geq 2$ and $s_{2} \geq 1$,

$$
\sum_{s_{1}, s_{3} \geq 2, s_{2} \geq 1} \mathbf{x}^{\mathbf{s}-\mathbf{1}} \zeta\left(s_{1}, s_{2}\right) \zeta\left(s_{3}\right)
$$

$$
\begin{gathered}
=\sum_{s_{1}, s_{3} \geq 2, s_{2} \geq 1} \mathbf{x}^{\mathbf{s}-\mathbf{1}} \sum_{\substack{t_{1} \geq 2, t_{2} \geq 1 \\
t_{1}+t_{2}=s_{1}+s_{3}}}\left[\begin{array}{l}
t_{1} \\
s_{3}
\end{array}\right] \zeta\left(t_{1}, t_{2}, s_{2}\right) \\
+\sum_{s_{1}, s_{3} \geq 2, s_{2} \geq 1} \mathbf{x}^{\mathbf{s}-\mathbf{1}} \sum_{\substack{t_{1} \geq 2, t_{2}, t_{3} \geq 1 \\
t_{1}+t_{2}+t_{3} \\
=s_{1}+s_{2}+s_{3}}}\left[\begin{array}{l}
t_{1} \\
s_{1}
\end{array}\right]\left(\begin{array}{c}
t_{2}-1 \\
s_{2}-t_{3}
\end{array}\right) \zeta\left(t_{1}, t_{2}, t_{3}\right) \\
+\sum_{s_{1}, s_{3} \geq 2, s_{2} \geq 1} \mathbf{x}^{\mathbf{s}-\mathbf{1}} \sum_{\substack{t_{1} \geq 2, t_{2}, t_{3} \geq 1 \\
t_{1}+t_{2}+t_{3} \\
=s_{1}+s_{2}+s_{3}}}\left[\begin{array}{l}
t_{1} \\
s_{1}
\end{array}\right]\left[\begin{array}{l}
t_{2} \\
s_{2}
\end{array}\right] \zeta\left(t_{1}, t_{2}, t_{3}\right) .
\end{gathered}
$$


Similar to the last section, we can get

$$
\begin{aligned}
(4.1)= & G_{3}\left(x_{13}, x_{1}, x_{2}\right)-G_{3}\left(x_{1}, x_{1}, x_{2}\right) \\
& -\sum_{s_{1} \geq 2, s_{2} \geq 1} x_{3}^{s_{1}-1} x_{2}^{s_{2}-1} \zeta\left(s_{1}, 1, s_{2}\right), \\
(4.2)= & G_{3}\left(x_{13}, x_{23}, x_{2}\right)-G_{3}\left(x_{3}, x_{23}, x_{2}\right)-G_{3}\left(x_{1}, x_{2}, x_{2}\right), \\
(4.3)= & G_{3}\left(x_{13}, x_{23}, x_{3}\right)-G_{3}\left(x_{3}, x_{23}, x_{3}\right) \\
& -\sum_{s_{1} \geq 2, s_{2} \geq 1} x_{1}^{s_{1}-1} x_{2}^{s_{2}-1} \zeta\left(s_{1}, s_{2}, 1\right) .
\end{aligned}
$$

On the other hand,

$$
\begin{aligned}
& \sum_{s_{1} \geq 2, s_{2} \geq 1, s_{3} \geq 2} \mathbf{x}^{\mathbf{s}-\mathbf{1}} \zeta\left(s_{1}, s_{3}, s_{2}\right) \\
= & G_{3}\left(x_{1}, x_{3}, x_{2}\right)-\sum_{s_{1} \geq 2, s_{2} \geq 1} x_{1}^{s_{1}-1} x_{2}^{s_{2}-1} \zeta\left(s_{1}, 1, s_{2}\right) \\
& \sum_{s_{1} \geq 2, s_{2} \geq 1, s_{3} \geq 2} \mathbf{x}^{\mathbf{s}-\mathbf{1}} \zeta\left(s_{3}, s_{1}, s_{2}\right) \\
= & G_{3}\left(x_{3}, x_{1}, x_{2}\right)-\sum_{s_{1} \geq 2, s_{2} \geq 1} x_{1}^{s_{1}-1} x_{2}^{s_{2}-1} \zeta\left(s_{1}, 1, s_{2}\right) \\
& \sum_{s_{1} \geq 2, s_{2} \geq 1, s_{3} \geq 2} \mathbf{x}^{\mathbf{s}-\mathbf{1}} \zeta\left(s_{1}, s_{2}, s_{3}\right) \\
= & G_{3}\left(x_{1}, x_{2}, x_{3}\right)-\sum_{s_{1} \geq 2, s_{2} \geq 1} x_{1}^{s_{1}-1} x_{2}^{s_{2}-1} \zeta\left(s_{1}, s_{2}, 1\right) .
\end{aligned}
$$

Also,

$$
\begin{aligned}
& \sum_{s_{1} \geq 2, s_{2} \geq 1, s_{3} \geq 2} \mathbf{x}^{\mathbf{s}-\mathbf{1}} \zeta\left(s_{1}+s_{3}, s_{2}\right) \\
= & \sum_{s_{2} \geq 1} x_{2}^{s_{2}-1} \sum_{s \geq 2}\left(\sum_{\substack{s_{1} \geq 2 \\
s_{3} \geq 2 \\
s_{1}+s_{3}=s}} x_{1}^{s_{1}-1} x_{3}^{s_{3}-1}\right) \zeta\left(s, s_{2}\right) \\
= & \sum_{s_{2} \geq 1} x_{2}^{s_{2}-1} \sum_{s \geq 2}\left(\frac{x_{3}^{s-1}-x_{1}^{s-1}}{x_{3}-x_{1}}-x_{3}^{s-2}-x_{1}^{s-2}+\delta_{s, 2}\right) \zeta\left(s, s_{2}\right) \\
= & \frac{G_{2}\left(x_{3}, x_{2}\right)-G_{2}\left(x_{1}, x_{2}\right)}{x_{3}-x_{1}}-\frac{G_{2}\left(x_{1}, x_{2}\right)}{x_{1}}
\end{aligned}
$$




$$
-\frac{G_{2}\left(x_{3}, x_{2}\right)}{x_{3}}+\sum_{s \geq 1} x_{2}^{s-1} \zeta(2, s),
$$

where $\delta_{s, 2}=1$ if $s=2$ and $\delta_{s, 2}=0$ otherwise. Similarly,

$$
\begin{aligned}
& \sum_{s_{1} \geq 2, s_{2} \geq 1, s_{3} \geq 2} \mathbf{x}^{\mathbf{s}-\mathbf{1}} \zeta\left(s_{1}, s_{2}+s_{3}\right) \\
= & \sum_{s_{1} \geq 2} x_{1}^{s_{1}-1} \sum_{s \geq 1}\left(\sum_{\substack{s_{2} \geq 1 \\
s_{3} \geq 2 \\
s_{2}+s_{3}=s}} x_{2}^{s_{2}-1} x_{3}^{s_{3}-1}\right) \zeta\left(s_{1}, s\right) \\
= & \sum_{s_{1} \geq 2} x_{1}^{s_{1}-1} \sum_{s \geq 1}\left(\frac{x_{3}^{s-1}-x_{2}^{s-1}}{x_{3}-x_{1}}-x_{2}^{s-2}+\delta_{s, 1} x_{2}^{-1}\right) \zeta\left(s_{1}, s\right) \\
= & \frac{G_{2}\left(x_{1}, x_{3}\right)-G_{2}\left(x_{1}, x_{2}\right)}{x_{3}-x_{2}}-\frac{G_{2}\left(x_{1}, x_{2}\right)}{x_{2}} \\
& +\frac{1}{x_{2}} \sum_{s \geq 2} x_{1}^{s-1} \zeta(s, 1) .
\end{aligned}
$$

Notice we have the stuffle relation

$$
\begin{aligned}
\zeta\left(s_{1}, s_{2}\right) \zeta\left(s_{3}\right)=\zeta\left(s_{1}, s_{2}, s_{3}\right)+\zeta\left(s_{1}, s_{3}, s_{2}\right) \\
\\
+\zeta\left(s_{3}, s_{1}, s_{2}\right)+\zeta\left(s_{1}+s_{3}, s_{2}\right)+\zeta\left(s_{1}, s_{2}+s_{3}\right) .
\end{aligned}
$$

Hence, the sum of (4.4)-(4.8) is equal to the sum of (4.1)-(4.3), and therefore, we obtain the following:

Theorem 4.1. We have

$$
\begin{aligned}
& G_{3}\left(x_{13}, x_{1}, x_{2}\right)-G_{3}\left(x_{1}, x_{1}, x_{2}\right)+G_{3}\left(x_{13}, x_{23}, x_{2}\right) \\
& \quad-G_{3}\left(x_{3}, x_{23}, x_{2}\right)-G_{3}\left(x_{1}, x_{2}, x_{2}\right) \\
& \quad+G_{3}\left(x_{13}, x_{23}, x_{3}\right)-G_{3}\left(x_{3}, x_{23}, x_{3}\right) \\
& \quad-G_{3}\left(x_{1}, x_{2}, x_{3}\right)-G_{3}\left(x_{1}, x_{3}, x_{2}\right) \\
& \quad-G_{3}\left(x_{3}, x_{1}, x_{2}\right) \\
& =\frac{G_{2}\left(x_{3}, x_{2}\right)-G_{2}\left(x_{1}, x_{2}\right)}{x_{3}-x_{1}}+\frac{G_{2}\left(x_{1}, x_{3}\right)-G_{2}\left(x_{1}, x_{2}\right)}{x_{3}-x_{2}} \\
& \quad-\frac{G_{2}\left(x_{1}, x_{2}\right)}{x_{1}}-\frac{G_{2}\left(x_{3}, x_{2}\right)}{x_{3}}-\frac{G_{2}\left(x_{1}, x_{2}\right)}{x_{2}}
\end{aligned}
$$




$$
\begin{aligned}
& -\sum_{s_{1} \geq 2, s_{2} \geq 1} x_{1}^{s_{1}-1} x_{2}^{s_{2}-1} \zeta\left(s_{1}, 1, s_{2}\right) \\
& +\sum_{s \geq 1} x_{2}^{s-1} \zeta(2, s)+\frac{1}{x_{2}} \sum_{s \geq 2} x_{1}^{s-1} \zeta(s, 1) .
\end{aligned}
$$

Proof. Clear.

Theorem 4.2. Let $a, b$ and $c$ be three real numbers. Then we have

$$
\begin{aligned}
& \sum_{\substack{j \geq 2, k, l \geq 1 \\
j+k+l=n}}\left[(a+c)^{j-1} a^{k} b^{l} c+a(a+c)^{j-1}(b+c)^{k-1}\left(c b^{l}+b c^{l}\right)\right. \\
& -a^{j+k-1} b^{l} c-a^{j} b^{k+l-1} c \\
& -a c^{j}(b+c)^{k-1}\left(b^{l}+b c^{l-1}\right) \\
& \left.-a^{j} b^{k} c^{l}-a^{j} c^{k} b^{l}-c^{j} a^{k} b^{l}\right] \zeta(j, k, l) \\
= & \sum_{j \geq 2, k \geq 1}\left[\frac{\left(a c^{j}-a^{j} c\right) b^{k}}{c-a}+\frac{a^{j}\left(b c^{k}-b^{k} c\right)}{c-b}\right. \\
& \left.-a^{j-1} b^{k} c-a b^{k} c^{j-1}-a^{j} b^{k-1} c\right] \zeta(j, k) \\
& -\sum_{k=2}^{n-2} a^{k} b^{n-1-k} c \zeta(k, 1, n-1-k) \\
& +a b^{n-2} c \zeta(2, n-2)+a^{n-1} c \zeta(n-1,1) .
\end{aligned}
$$

Proof. Multiplying $x_{1} x_{2} x_{3}$ on (4.9), taking $x_{1}=a t, x_{2}=b t$ and $x_{3}=c t$ and then comparing the coefficients of $t^{n}$, we arrive at (4.10) immediately.

Notice that Theorem 4.1 is very similar to [9, Theorem 1.1 (i)] but not the same. Moreover, by comparing the two results, we immediately obtain the following.

Theorem 4.3. We have

$$
G_{3}\left(x_{1}, x_{1}, x_{2}\right)+G_{3}\left(x_{1}, x_{2}, x_{2}\right)-\frac{G_{2}\left(x_{1}, x_{2}\right)}{x_{1}}-\frac{G_{2}\left(x_{1}, x_{2}\right)}{x_{2}}
$$




$$
\begin{aligned}
=\sum_{s_{1} \geq 2, s_{2} \geq 1} x_{1}^{s_{1}-1} x_{2}^{s_{2}-1} \zeta\left(s_{1}, 1, s_{2}\right)- & \sum_{s \geq 1} x_{2}^{s-1} \zeta(2, s) \\
& -\frac{1}{x_{2}} \sum_{s \geq 2} x_{1}^{s-1} \zeta(s, 1) .
\end{aligned}
$$

Proof. The terms appearing in (4.11) are exactly those that are in Theorem 4.1 but not in [9, Theorem 1.1 (i)].

Theorem 4.4. Let $a$ and $b$ be two real numbers. Then we have

$$
\begin{aligned}
& \sum_{\substack{j \geq 2, k, l \geq 1 \\
j+k+l=n}}\left[a^{j+k-1} b^{l}+a^{j} b^{k+l-1}\right] \zeta(j, k, l) \\
& -\sum_{\substack{j \geq 2, k \geq 1 \\
j+k=n}}\left[a^{j-1} b^{k}+a^{j} b^{k-1}\right] \zeta(j, k) \\
= & \sum_{k=2}^{n-2} a^{k} b^{n-1-k} \zeta(k, 1, n-1-k) \\
& -a b^{n-2} \zeta(2, n-2)-a^{n-1} \zeta(n-1,1) .
\end{aligned}
$$

Proof. This follows from Theorem 4.3 immediately.

Corollary 4.5. Let $n$ be a positive integer such that $n \geq 3$. Then

$$
\begin{aligned}
& \sum_{\substack{j \geq 2, k, l \geq 1 \\
j+k+l=n}}(2 j+k) \zeta(j, k, l)- \sum_{\substack{k \geq 2, l \geq 1 \\
k+l=n-1}} k \zeta(k, 1, l) \\
&=\zeta(2, n-2)+4 \zeta(n)-(3 n-5) \zeta(n-1,1) .
\end{aligned}
$$

Proof. Differentiating (4.12) with respect to $a$ and then putting $a=b=1$, we get

$$
\begin{gathered}
\sum_{\substack{j \geq 2, k, l \geq 1 \\
j+k+l=n}}(2 j+k-1) \zeta(j, k, l)-\sum_{\substack{j \geq 2, k \geq 1 \\
j+k=n}}(2 j-1) \zeta(j, k) \\
=\sum_{\substack{k \geq 2, l \geq 1 \\
k+l=n-1}} k \zeta(k, 1, l)-\zeta(2, n-2)-(n-1) \zeta(n-1,1) .
\end{gathered}
$$


The corollary now follows from (2.3) and the sum formulas (2.2) for $d=2,3$.

The following corollary provides a sum formula relating some special type triple zeta and double zeta values. It also appeared in [9] as (5.12) which is a special case of [6, Theorem 5.1].

Corollary 4.6. Let $n$ be a positive integer such that $n \geq 3$. Then

$$
\sum_{k=2}^{n-1} \zeta(k, 1, n-k)=\zeta(2, n-1)+\zeta(n, 1) .
$$

Proof. Taking $a=b=1$ and letting $c \rightarrow 1$ in Theorem 4.2, we get

$$
\begin{aligned}
& \sum_{\substack{j \geq 2, k, l \geq 1 \\
j+k+l=n}}\left(2^{j-1}+2^{j+k-1}+2^{k}-5\right) \zeta(j, k, l) \\
& -(n-5) \sum_{\substack{j \geq 2, k \geq 1 \\
j+k=n}} \zeta(j, k) \\
= & -\sum_{\substack{k=2 \\
n-2}(k, 1, n-1-k)} \zeta(n-1,1) .
\end{aligned}
$$

By sum formula (2.2), we see that

$$
\sum_{\substack{j \geq 2, k \geq 1 \\ j+k=n}} \zeta(j, k)=\sum_{\substack{j \geq 2, k, l \geq 1 \\ j+k+l=n}} \zeta(j, k, l)=\zeta(n) .
$$

Now (4.14) quickly follows from the weighted sum formula of Guo and Xie [5, Theorem 1.1] (setting $k=2$ there).

5. Depth 4: Product of two double zetas. In this last section, we turn our attention to the depth 4 case and derive some new families of MZV identities using the idea of generating functions developed as above. Throughout this section, we set $\mathbf{x}^{\mathbf{s}-\mathbf{1}}=x_{1}^{s_{1}-1} x_{2}^{s_{2}-1} x_{3}^{s_{3}-1} x_{4}^{s_{4}-1}$. We also use the short hand $t_{i j}=t_{i}+t_{j}, s_{i j}=s_{i}+s_{j}$, and so on. 
First, for the integers $s_{1}, s_{3} \geq 2$ and $s_{2}, s_{4} \geq 1$, Guo and Xie proved the following at the end of [5]:

$$
\begin{aligned}
& \zeta\left(s_{1}, s_{2}\right) \zeta\left(s_{3}, s_{4}\right) \\
& =\sum_{\substack{t_{1} \geq 2, t_{2}, t_{3} \geq 1 \\
t_{123}=s_{123}}}\left[\begin{array}{l}
t_{1} \\
s_{1}
\end{array}\right]\left[\begin{array}{l}
t_{2} \\
s_{2}
\end{array}\right] \zeta\left(t_{1}, t_{2}, t_{3}, s_{4}\right) \\
& +\sum_{\substack{t_{1} \geq 2, t_{2}, t_{3} \geq 1 \\
t_{123}=s_{134}}}\left[\begin{array}{l}
t_{1} \\
s_{3}
\end{array}\right]\left[\begin{array}{l}
t_{2} \\
s_{4}
\end{array}\right] \zeta\left(t_{1}, t_{2}, t_{3}, s_{2}\right) \\
& +\sum_{\substack{t_{1} \geq 2, t_{2}, t_{3}, t_{4} \geq 1 \\
t_{1234}=s_{1234}}}\left[\left[\begin{array}{l}
t_{1} \\
s_{1}
\end{array}\right]\left(\begin{array}{c}
t_{2}-1 \\
t_{12}-s_{13}
\end{array}\right)\left(\left(\begin{array}{c}
t_{3}-1 \\
s_{4}-t_{4}
\end{array}\right)+\left[\begin{array}{l}
t_{3} \\
s_{4}
\end{array}\right]\right)\right. \\
& \left.+\underset{\left.\begin{array}{l}
t_{1} \\
s_{3}
\end{array}\right]}{ }\left(\begin{array}{c}
t_{2}-1 \\
t_{12}-s_{13}
\end{array}\right)\left(\left(\begin{array}{c}
t_{3}-1 \\
s_{2}-t_{4}
\end{array}\right)+\left[\begin{array}{l}
t_{3} \\
s_{2}
\end{array}\right]\right)\right] \zeta\left(t_{1}, t_{2}, t_{3}, t_{4}\right) .
\end{aligned}
$$

Hence,

$$
\sum_{\substack{s_{1}, s_{3} \geq 2 \\ s_{2}, s_{4} \geq 1}} \mathbf{x}^{\mathbf{s}-\mathbf{1}} \zeta\left(s_{1}, s_{2}\right) \zeta\left(s_{3}, s_{4}\right)
$$

$$
=\sum_{\substack{s_{1}, s_{3} \geq 2 \\
s_{2}, s_{4} \geq 1}} \mathbf{x}^{\mathbf{s}-\mathbf{1}} \sum_{\substack{t_{1} \geq 2, t_{2}, t_{3} \geq 1 \\
t_{123}=s_{123}}}\left[\begin{array}{l}
t_{1} \\
s_{1}
\end{array}\right]\left[\begin{array}{l}
t_{2} \\
s_{2}
\end{array}\right] \zeta\left(t_{1}, t_{2}, t_{3}, s_{4}\right)
$$

$$
\begin{aligned}
& +\sum_{\substack{s_{1}, s_{3} \geq 2 \\
s_{2}, s_{4} \geq 1}} \mathbf{x}^{\mathbf{s}-\mathbf{1}} \sum_{\substack{t_{1} \geq 2, t_{2}, t_{3} \geq 1 \\
t_{123}=s_{134}}}\left[\begin{array}{l}
t_{1} \\
s_{3}
\end{array}\right]\left[\begin{array}{l}
t_{2} \\
s_{4}
\end{array}\right] \zeta\left(t_{1}, t_{2}, t_{3}, s_{2}\right) \\
& +\sum_{\substack{s_{1}, s_{3} \geq 2 \\
s_{2}, s_{4} \geq 1}} \mathbf{x}^{\mathbf{s}-\mathbf{1}} \sum_{\substack{t_{1} \geq 2, t_{2}, t_{3}, t_{4} \geq 1 \\
t_{1234}=s_{1234}}}\left[\begin{array}{l}
t_{1} \\
s_{1}
\end{array}\right]
\end{aligned}
$$

$$
\begin{aligned}
& \cdot\left(\begin{array}{c}
t_{2}-1 \\
t_{12}-s_{13}
\end{array}\right)\left(\begin{array}{c}
t_{3}-1 \\
s_{4}-t_{4}
\end{array}\right) \zeta\left(t_{1}, t_{2}, t_{3}, t_{4}\right) \\
& +\sum_{\substack{s_{1}, s_{3} \geq 2 \\
s_{2}, s_{4} \geq 1}} \mathbf{x}^{\mathbf{s}-\mathbf{1}} \sum_{\substack{t_{1} \geq 2, t_{2}, t_{3}, t_{4} \geq 1 \\
t_{1234}=s_{1234}}}\left[\begin{array}{l}
t_{1} \\
s_{1}
\end{array}\right]
\end{aligned}
$$$$
\cdot\left(\begin{array}{c}
t_{2}-1 \\
t_{12}-s_{13}
\end{array}\right)\left[\begin{array}{l}
t_{3} \\
s_{4}
\end{array}\right] \zeta\left(t_{1}, t_{2}, t_{3}, t_{4}\right)
$$ 


$$
+\sum_{\substack{s_{1}, s_{3} \geq 2 \\
s_{2}, s_{4} \geq 1}} \mathbf{x}^{\mathbf{s}-\mathbf{1}} \sum_{\substack{t_{1} \geq 2, t_{2}, t_{3}, t_{4} \geq 1 \\
t_{1234}=1 \\
s_{1234}}}\left[\begin{array}{l}
t_{1} \\
s_{3}
\end{array}\right]\left(\begin{array}{c}
t_{2}-1 \\
t_{12}-s_{13}
\end{array}\right)
$$

$$
\begin{aligned}
& \cdot\left(\begin{array}{c}
t_{3}-1 \\
s_{2}-t_{4}
\end{array}\right) \zeta\left(t_{1}, t_{2}, t_{3}, t_{4}\right) \\
& +\sum_{\substack{s_{1}, s_{3} \geq 2 \\
s_{2}, s_{4} \geq 1}} \mathbf{x}^{\mathbf{s}-\mathbf{1}} \sum_{\substack{t_{1} \geq 2, t_{2}, t_{3}, t_{4} \geq 1 \\
t_{1234}=s_{1234}}}\left[\begin{array}{l}
t_{1} \\
s_{3}
\end{array}\right]\left(\begin{array}{c}
t_{2}-1 \\
t_{12}-s_{13}
\end{array}\right) \\
& \cdot\left[\begin{array}{l}
t_{3} \\
s_{2}
\end{array}\right] \zeta\left(t_{1}, t_{2}, t_{3}, t_{4}\right) .
\end{aligned}
$$

As before, we can get

$$
\begin{aligned}
(5.1)= & G_{4}\left(x_{13}, x_{23}, x_{3}, x_{4}\right)-G_{4}\left(x_{3}, x_{23}, x_{3}, x_{4}\right) \\
& -\sum_{\substack{s_{1} \geq 2 \\
s_{2}, s_{4} \geq 1}} x_{1}^{s_{1}-1} x_{2}^{s_{2}-1} x_{4}^{s_{4}-1} \zeta\left(s_{1}, s_{2}, 1, s_{4}\right), \\
(5.3)= & G\left(x_{13}, x_{23}, x_{24}, x_{4}\right)-G\left(x_{3}, x_{23}, x_{24}, x_{4}\right)-G\left(x_{1}, x_{2}, x_{24}, x_{4}\right), \\
(5.4)= & G\left(x_{13}, x_{23}, x_{24}, x_{2}\right)-G\left(x_{3}, x_{23}, x_{24}, x_{2}\right)-G\left(x_{1}, x_{2}, x_{24}, x_{2}\right), \\
(5.2)= & \sigma_{x_{1}, x_{3}} \sigma_{x_{2}, x_{4}}(5.1), \quad(5.5)=\sigma_{x_{1}, x_{3}} \sigma_{x_{2}, x_{4}}(5.3), \\
(5.6)= & \sigma_{x_{1}, x_{3}} \sigma_{x_{2}, x_{4}}(5.4) .
\end{aligned}
$$

On the other hand, by the stuffle relation

$$
\begin{aligned}
& \zeta\left(s_{1}, s_{2}\right) \zeta\left(s_{3}, s_{4}\right)=\zeta\left(s_{3}, s_{4}, s_{1}, s_{2}\right)+\zeta\left(s_{3}, s_{1}, s_{4}, s_{2}\right) \\
& \quad+\zeta\left(s_{1}, s_{3}, s_{4}, s_{2}\right)+\zeta\left(s_{1}, s_{3}, s_{2}, s_{4}\right)+\zeta\left(s_{1}, s_{2}, s_{3}, s_{4}\right) \\
& \quad+\zeta\left(s_{3}, s_{1}, s_{2}, s_{4}\right)+\zeta\left(s_{13}, s_{2}, s_{4}\right) \\
& \quad+\zeta\left(s_{1}, s_{23}, s_{4}\right)+\zeta\left(s_{13}, s_{2}, s_{4}\right) \\
& \quad+\zeta\left(s_{3}, s_{14}, s_{2}\right)+\zeta\left(s_{1}, s_{3}, s_{24}\right) \\
& \quad+\zeta\left(s_{3}, s_{1}, s_{24}\right)+\zeta\left(s_{13}, s_{24}\right)
\end{aligned}
$$

Thus, we have

$$
\sum_{\substack{s_{1}, s_{3} \geq 2 \\ s_{2}, s_{4} \geq 1}} \mathbf{x}^{\mathbf{s}-\mathbf{1}} \zeta\left(s_{1}, s_{2}\right) \zeta\left(s_{3}, s_{4}\right)
$$




$$
\begin{aligned}
& =\sum_{\substack{s_{1}, s_{3} \geq 2 \\
s_{2}, s_{4} \geq 1}} \mathbf{x}^{\mathbf{s}-\mathbf{1}} \zeta\left(s_{3}, s_{4}, s_{1}, s_{2}\right)+\sum_{\substack{s_{1}, s_{3} \geq 2 \\
s_{2}, s_{4} \geq 1}} \mathbf{x}^{\mathbf{s}-\mathbf{1}} \zeta\left(s_{3}, s_{1}, s_{4}, s_{2}\right) \\
& +\sum_{\substack{s_{1}, s_{3} \geq 2 \\
s_{2}, s_{4} \geq 1}} \mathbf{x}^{\mathbf{s}-\mathbf{1}} \zeta\left(s_{1}, s_{3}, s_{4}, s_{2}\right)+\sum_{\substack{s_{1}, s_{3} \geq 2 \\
s_{2}, s_{4} \geq 1}} \mathbf{x}^{\mathbf{s}-\mathbf{1}} \zeta\left(s_{1}, s_{3}, s_{2}, s_{4}\right) \\
& +\sum_{\substack{s_{1}, s_{3} \geq 2 \\
s_{2}, s_{4} \geq 1}} \mathbf{x}^{\mathbf{s}-\mathbf{1}} \zeta\left(s_{1}, s_{2}, s_{3}, s_{4}\right)+\sum_{\substack{s_{1}, s_{3} \geq 2 \\
s_{2}, s_{4} \geq 1}} \mathbf{x}^{\mathbf{s}-\mathbf{1}} \zeta\left(s_{3}, s_{1}, s_{2}, s_{4}\right) \\
& +\sum_{\substack{s_{1}, s_{3} \geq 2 \\
s_{2}, s_{4} \geq 1}} \mathbf{x}^{\mathbf{s}-\mathbf{1}} \zeta\left(s_{13}, s_{4}, s_{2}\right)+\sum_{\substack{s_{1}, s_{3} \geq 2 \\
s_{2}, s_{4} \geq 1}} \mathbf{x}^{\mathbf{s}-\mathbf{1}} \zeta\left(s_{1}, s_{23}, s_{4}\right) \\
& +\sum_{\substack{s_{1}, s_{3} \geq 2 \\
s_{2}, s_{4} \geq 1}} \mathbf{x}^{\mathbf{s}-\mathbf{1}} \zeta\left(s_{13}, s_{2}, s_{4}\right)+\sum_{\substack{s_{1}, s_{3} \geq 2 \\
s_{2}, s_{4} \geq 1}} \mathbf{x}^{\mathbf{s}-\mathbf{1}} \zeta\left(s_{3}, s_{4}+s_{1}, s_{2}\right) \\
& +\sum_{\substack{s_{1}, s_{3} \geq 2 \\
s_{2}, s_{4} \geq 1}} \mathbf{x}^{\mathbf{s}-\mathbf{1}} \zeta\left(s_{1}, s_{3}, s_{24}\right)+\sum_{\substack{s_{1}, s_{3} \geq 2 \\
s_{2}, s_{4} \geq 1}} \mathbf{x}^{\mathbf{s}-\mathbf{1}} \zeta\left(s_{3}, s_{1}, s_{24}\right) \\
& +\sum_{\substack{s_{1}, s_{3} \geq 2 \\
s_{2}, s_{4} \geq 1}} \mathbf{x}^{\mathbf{s}-\mathbf{1}} \zeta\left(s_{13}, s_{24}\right)
\end{aligned}
$$

One can verify the following identities as before:

$$
\begin{aligned}
(5.7)= & G_{4}\left(x_{3}, x_{4}, x_{1}, x_{2}\right)+G_{4}\left(x_{3}, x_{1}, x_{4}, x_{2}\right) \\
& -\sum_{\substack{s_{3} \geq 2 \\
s_{2}, s_{4} \geq 1}} x_{2}^{s_{2}-1} x_{3}^{s_{3}-1} x_{4}^{s_{4}-1} \zeta\left(s_{3}, s_{4}, 1, s_{2}\right) \\
& -\sum_{\substack{s_{3} \geq 2 \\
s_{2}, s_{4} \geq 1}} x_{2}^{s_{2}-1} x_{3}^{s_{3}-1} x_{4}^{s_{4}-1} \zeta\left(s_{3}, 1, s_{4}, s_{2}\right) \\
(5.8)= & G_{4}\left(x_{1}, x_{3}, x_{4}, x_{2}\right)+G_{4}\left(x_{1}, x_{3}, x_{2}, x_{4}\right) \\
& -\sum_{\substack{s_{1} \geq 2 \\
s_{2}, s_{4} \geq 1}} x_{1}^{s_{1}-1} x_{2}^{s_{2}-1} x_{4}^{s_{4}-1} \zeta\left(s_{1}, 1, s_{4}, s_{2}\right) \\
& -\sum_{\substack{s_{1} \geq 2 \\
s_{2}, s_{4} \geq 1}} x_{1}^{s_{1}-1} x_{2}^{s_{2}-1} x_{4}^{s_{4}-1} \zeta\left(s_{1}, 1, s_{2}, s_{4}\right) \\
(5.9)= & G_{4}\left(x_{1}, x_{2}, x_{3}, x_{4}\right)+G_{4}\left(x_{3}, x_{1}, x_{2}, x_{4}\right)
\end{aligned}
$$




$$
\begin{aligned}
& -\sum_{\substack{s_{1} \geq 2 \\
s_{2}, s_{4} \geq 1}} x_{1}^{s_{1}-1} x_{2}^{s_{2}-1} x_{4}^{s_{4}-1} \zeta\left(s_{1}, s_{2}, 1, s_{4}\right) \\
& -\sum_{\substack{s_{3} \geq 2 \\
s_{2}, s_{4} \geq 1}} x_{2}^{s_{2}-1} x_{3}^{s_{3}-1} x_{4}^{s_{4}-1} \zeta\left(s_{3}, 1, s_{2}, s_{4}\right) \\
& (5.10)=\bigoplus_{\mathcal{C}\left(x_{1}, x_{3}\right)}\left\{\frac{G_{3}\left(x_{1}, x_{4}, x_{2}\right)}{x_{1}-x_{3}}-\frac{G_{3}\left(x_{1}, x_{4}, x_{2}\right)}{x_{1}}\right\} \\
& +\frac{G_{3}\left(x_{1}, x_{2}, x_{4}\right)-G_{3}\left(x_{1}, x_{3}, x_{4}\right)}{x_{2}-x_{3}}-\frac{G_{3}\left(x_{1}, x_{2}, x_{4}\right)}{x_{2}} \\
& +\frac{1}{x_{2}} \sum_{\substack{s \geq 2 \\
t \geq 1}} x_{1}^{s-1} x_{4}^{t-1} \zeta(s, 1, t)+\sum_{s, t \geq 1} x_{2}^{s-1} x_{4}^{t-1} \zeta(2, t, s) \\
& (5.11)=\sigma_{x_{1}, x_{3}} \sigma_{x_{2}, x_{4}}(5.10) \\
& (5.12)=\bigoplus_{\mathcal{C}\left(x_{2}, x_{4}\right)}\left\{\bigoplus _ { \mathcal { C } ( x _ { 1 } , x _ { 3 } ) } \left\{\frac{G_{3}\left(x_{1}, x_{3}, x_{2}\right)}{x_{2}-x_{4}}\right.\right. \\
& \left.\left.-\frac{1}{x_{2}-x_{4}} \sum_{\substack{t \geq 2 \\
s \geq 1}} x_{1}^{t-1} x_{2}^{s-1} \zeta(t, 1, s)\right\}\right\} \\
& (5.13)=\bigoplus_{\mathcal{C}\left(x_{2}, x_{4}\right)}\left\{\bigoplus_{\mathcal{C}\left(x_{1}, x_{3}\right)}\left\{\left(\frac{1}{x_{1}-x_{3}}-\frac{1}{x_{1}}\right) \frac{G_{2}\left(x_{1}, x_{2}\right)}{x_{2}-x_{4}}\right\}\right. \\
& \left.+\frac{1}{x_{2}-x_{4}} \sum_{t \geq 1} x_{2}^{t-1} \zeta(2, t)\right\} \text {. }
\end{aligned}
$$

Hence, the sum of (5.1)-(5.6) is equal to the sum of (5.7)-(5.13). This equality establishes an identity involving $G_{4}$ and four formal variables $x_{1}, \ldots, x_{4}$. By taking $x_{1}=a t, x_{2}=b t, x_{3}=c t$ and $x_{4}=d t$ and comparing the coefficient of $t^{n-4}$, we can obtain our last theorem.

Let $S_{1}=\left\{e, \sigma_{a, c} \sigma_{b, d}\right\}$, where $\sigma_{a, c}$ (respectively, $\left.\sigma_{b, d}\right)$ denotes the transposition that switches $a$ and $c$ (respectively, $b$ and $d$ ) and

$$
S_{2}=\left\{e, \sigma_{a, c} \sigma_{b, d}, \sigma_{a, c}, \sigma_{b, c}, \sigma_{b, d}, \sigma_{c, d, b, a}\right\} .
$$

For any subset $S$ of the symmetric group $\mathfrak{S}_{4}$, let

$$
\bigoplus_{S} f(a, b, c, d)=\sum_{\sigma \in S} f(\sigma(a), \sigma(b), \sigma(c), \sigma(d)) .
$$


Theorem 5.1. Let $a, b, c$ and $d$ be any real numbers. Then, for any positive integer $n \geq 2$, we have

$$
\begin{aligned}
& \bigoplus_{S_{1}}\left\{\sum_{\substack{i \geq 2 \\
i+j+k+l=n}}\left[a b c(a+c)^{i-1}(b+c)^{j-1}-a b c^{i}(b+c)^{j-1}-c a^{i} b^{j}\right]\right. \\
& \cdot(b+d)^{k-1} d^{l} \cdot \zeta(i, j, k, l) \\
& +\sum_{\substack{i \geq 2 \\
i+j+\bar{k}+l=n}}\left[(a+c)^{i-1}-c^{i-1}\right] a b(b+c)^{j-1} c^{k} d^{l} \zeta(i, j, k, l) \\
& +\sum_{\substack{i \geq 2 \\
i+j+l=n-1}}\left(b^{j} d^{l}+d^{j} b^{l}\right) c a^{i} \zeta(i, 1, j, l) \\
& +\sum_{\substack{i \geq 2 \\
i+j+\bar{k}+l=n}}\left[a c d(a+c)^{i-1}(b+c)^{j-1}-a d c^{i}(b+c)^{j-1}-c d a^{i} b^{j-1}\right] \\
& \left.\cdot(b+d)^{k-1} b^{l} \cdot \zeta(i, j, k, l)\right\} \\
& =\bigoplus_{S_{2}}\left\{\sum_{\substack{i \geq 2 \\
i+j+k+l=n}} a^{i} b^{j} c^{k} d^{l} \zeta(i, j, k, l)\right\} \\
& +\bigoplus_{S_{1}}\left\{\sum_{\substack{i \geq 2 \\
i+j+k=n}}\left(\frac{c b^{j}-b c^{j}}{b-c}-c b^{j-1}\right) a^{i} d^{k} \zeta(i, j, k)\right. \\
& +\sum_{\substack{i \geq 2 \\
i+j+k=n}}\left(\frac{c a^{i}-a c^{i}}{a-c}-c a^{i-1}-a c^{i-1}\right) b^{j} d^{k} \zeta(i, j, k) \\
& +\sum_{\substack{i \geq 2 \\
i+j+k=n}} \frac{d b^{k}-b d^{k}}{b-d} a^{i} c^{j} \zeta(i, j, k) \\
& +\sum_{j+k=n-2} a c d^{j} b^{k} \zeta(2, j, k)+\sum_{\substack{i \geq 2 \\
i+k=n-1}} c a^{i} d^{k} \zeta(i, 1, k) \\
& \left.-\sum_{\substack{i \geq 2 \\
i+k=n-1}} \frac{d b^{k}-b d^{k}}{b-d} a^{i} c \zeta(i, 1, k)\right\}
\end{aligned}
$$




$$
\begin{aligned}
& +\sum_{\substack{i \geq 2, i+j=n}}\left(\frac{c a^{i}-a c^{i}}{a-c}-c a^{i-1}-a c^{i-1}\right) \frac{d b^{j}-b d^{j}}{b-d} \zeta(i, j) \\
& +\frac{a c\left(d b^{n-2}-b d^{n-2}\right)}{b-d} \zeta(2, n-2) .
\end{aligned}
$$

Corollary 5.2. Let $n \geq 5$ be any positive integer. Then

$$
\begin{gathered}
\text { (5.14) } 2 \sum_{\substack{i \geq 2 \\
i+j+l=n-1}} \zeta(i, 1, j, l)-\sum_{\substack{i \geq 2 \\
i+j+k+l=n}}\left(2^{i-1}+2^{k}\right) \zeta(i, j, k, l) \\
-\sum_{\substack{k \geq 2 \\
k+j=n-1}} k \zeta(k, 1, j) \\
=2 \zeta(2, n-2)+(n-3) \zeta(n-2,2)-(2 n-5) \zeta(n-1,1)+\frac{n+5}{2} \zeta(n) .
\end{gathered}
$$

Proof. Let $a=1=b$ and $c \rightarrow 1$ and $d \rightarrow 1$ in Theorem 5.1. Then we get

$$
\begin{aligned}
& 2 \sum_{\substack{i \geq 2 \\
i+j+k+l=n}}\left(2^{i+j+k-2}-2^{j+k-1}-2^{k}+2^{i+j-2}-2^{j-1}\right) \zeta(i, j, k, l) \\
& =6 \sum_{\substack{i \geq 2 \\
i+j+k+l=n}} \zeta(i, j, k, l)-4 \sum_{\substack{i \geq 2 \\
i+j+l=n-1}} \zeta(i, 1, j, l) \\
& +2 \sum_{\substack{i \geq 2 \\
i+j+k=n}}(n-6) \zeta(i, j, k) \\
& -2 \sum_{\substack{i \geq 2 \\
i+k=n-1}}(k-2) \zeta(i, 1, k) \\
& +2 \sum_{\substack{j+k=n-2 \\
+}} \zeta(2, j, k) \\
& +\sum_{\substack{i \geq 2 \\
i+j=n}}(i-3)(j-1) \zeta(i, j)+(n-3) \zeta(2, n-2) .
\end{aligned}
$$

By the sum formula and the weighted sum formula of Guo and Xie [5, 
Theorem 1.1] (setting $k=4$ there), we get:

$$
\begin{aligned}
& 2 \sum_{\substack{i \geq 2 \\
i+j+l=n-1}} \zeta(i, 1, j, l)-\sum_{\substack{i \geq 2 \\
i+j+k+l=n}}\left(2^{i-1}+2^{k}\right) \zeta(i, j, k, l) \\
& =\sum_{j+k=n-2} \zeta(2, j, k)-3 \zeta(n)-\sum_{i=2}^{n-2}(n-i-3) \zeta(i, 1, n-i-1) \\
& +\frac{1}{2} \sum_{i=2}^{n-1}(i-3)(n-i-1) \zeta(i, n-i) \\
& \quad+\frac{n-3}{2} \zeta(2, n-2) .
\end{aligned}
$$

Taking $l=2, i_{1}=2$ and $i_{2}=n-3$ in [6, Theorem 5.1], we get

$$
\sum_{j+k=n-2} \zeta(2, j, k)=\zeta(3, n-3)+\zeta(2, n-2) .
$$

Combining this with (2.3), (2.4), (3.14) and the sum formula (2.1), we see easily that (5.15) can be simplified to (5.14). This finishes the proof of the corollary.

Acknowledgments. Both authors would like to thank the Morningside Center of Mathematics, Chinese Academy of Science for hospitality when the paper was prepared.

\section{REFERENCES}

1. M. Eie, W. Liaw and Y. Ong, A restricted sum formula among multiple zeta values, J. Num. Theor. 129 (2009), 908-921.

2. L. Euler, Meditationes circa singulare serierum genus, Novi Comm. Acad. Sci. Petropol. 20 (1775), 140-186; reprinted in Opera Omnia 15, B. Teubner, Berlin, 1927.

3. H. Gangl, M. Kaneko and D. Zagier, Double zeta values and modular forms, in Automorphic forms and zeta functions, S. Böcherer et al., eds., World Scientific, Hackensack, NJ, 2006.

4. A. Granville, A decomposition of Riemanns zeta-function, in Analytic number theory, Y. Motohashi, ed., Lond. Math. Soc. Lect. Note 247, Cambridge, 1997.

5. L. Guo and B. Xie, Weighted sum formula for multiple zeta values, J. Num. Theor. 129 (2009), 2747-2765.

6. M.E. Hoffman, Multiple harmonic series, Pac. J. Math. 152 (1992), 275-290. 
7. K. Ihara, M. Kaneko and D. Zagier, Derivation and double shuffle relations for multiple zeta values, Compos. Math. 142 (2006), 307-338.

8. T. Machide, Weighted sums with two parameters of multiple zeta values and their formulas, Int. J. Num. Theor. 8 (2012), 1903-1921.

9. _ Extended double shuffle relations and the generating function of triple zeta values of any fixed weight, Kyushu Math. J. 67 (2013), 281-307.

10. Y. Ohno and W. Zudilin, Zeta stars, Comm. Num. Theor. Phys. 2 (2008), $325-347$.

11. D. Zagier, Values of zeta functions and their applications, in First european congress of mathematics, A. Joseph et al., eds., Birkhäuser, Basel, 1994.

Department of Mathematics, York College of Pennsylvania, York, PA 17403

Email address: hyuan@ycp.edu

ICMAT, C/Nicolás Cabrera, No. 13-15, 28049 Madrid, Spain

Email address: zhaoj@ihes.fr 